\title{
Cartan Frame Analysis of Hearts with Infarcts
}

\author{
Damien Goblot \\ Master of Computer Science \\ School of Computer Science \\ McGill University \\ Montréal,Quebec \\ 2016-08-13
}

A thesis submitted to McGill University in partial fulfillment of the requirements for the degree of Master of Science in Computer Science

(C) 2016 Damien Goblot 


\section{DEDICATION}

I would like to dedicate this thesis to my family who have always supported me and my close friends who continue to be a great source of inspiration. 


\section{ACKNOWLEDGEMENTS}

I would like to give my special and sincere thanks to Prof Kaleem Siddiqi who believed in my potential from the beginning and helped me get to McGill. I really appreciated how understanding he was when I had to face some health issues.

Mostly I would like to thank him for introducing me to this field and getting me to work with great and smart people in his network, as this is how I met my first post-graduation employer to name one of the many exciting opportunities I had in the course of my research. I am grateful to my examiner, Prof. Louis Collins, for many helpful comments and suggestions. These have improved the presentation of ideas in this thesis.

I would also sincerely like to thank Emmanuel Piuze for sharing with me his experience and work in Kaleem's lab and making me want to work in this environment and on an extension of his work. He put a lot of effort which allowed me to quickly

get up to speed and several of the experiments I carried out were facilitated by the algorithms and software implementations he had put into place.

Many thanks go to Mihaela Pop for being so supportive and available on all the projects that we worked on, and of course for the data she was kind enough to share with us to run our experiments on.

I would also like to thank Peter Savadjiev for helping me and clarifying obscure things on many occasions, Ryan Eckbo for sharing his precious knowledge and his help, and finally Babak Samari and Morteza Rezanejad who were so nice and welcoming in the lab. 


\begin{abstract}
This thesis uses a frame fitting method developed in [25] to describe the fiber geometry of mammalian hearts. Extending previous work, we analyze on a quantitative and qualitative level the extra information that this geometrical analysis can provide on infarcted hearts. Such hearts, right after the occurrence of an infarct, see the damaged region get filled with collagen and significantly lose contractile efficiency due to the loss of many fibers that were involved in the contraction during the beat cycle. General knowledge from Apparent Diffusion Coefficient (ADC) and Fractional Anisotropy (FA) map analysis does not provide an indication of how some surviving fibers can persist in the infarct region and how the remodeling process might still allow some contractile function, although hindered and modified by the geometrical reorganization and the decreased connectivity between regions remote from the infarct and potential surviving fibers in the infarct region. Our connection form parameter calculation and frame fitting error plots allow us to visualize regions mostly filled with collagen within the infarct region, as well as regions where some fiber structure still shows up, demonstrating post infarct contractile potential. Our approach could be a promising way to understand the process of remodeling of the heart wall following an infarct. This could also highlight the new geometrical structure and its relation to the unfortunately frequent secondary effects: tachycardia, arrhythmia and related abnormalities.
\end{abstract}




\begin{abstract}
ABRÉGÉ
Ce mémoire se base sur une méthode d'ajustement développée dans [25] pour décrire la géométrie des fibres du coeur de mammifères. En approfondissant le travail auparavant effectué, nous analisons au niveau qualitatif et quantitatif les informations supplémentaires que nous apportent cette analise géométrique réalisée sur des coeurs avec infarctus. Ces coeurs, juste après avoir subi un infarct, ont la région touchée par l'infarct qui se remplit de collagène et devient moins contractile de manière significative. En effet beaucoup des fibres impliquées dans la contraction pendant le cycle de battement du coeur sont mortes lors de l'infarct. Une connaissance générale délivrée par l'analise des cartographies d'anisotropie fractionnelle (FA) et de coefficient de diffusion apparent (ADC) ne donne aucune information sur d'éventuelles fibres qui auraient survi à l'infarct et dans quelle mesure elles peuvent toujours jouer un rôle dans la fonction contractile même si celle-ci est gravement aggravée et modifiée par la réorganisation géométrique des fibres et la moindre connectivitée entre les régions éloignées de l’infarct et les quelques régions dans la région de l'infarct qui y ont survécu. Notre calcul de coefficients de connexions affines de Cartan et nos représentations des erreurs d'ajustement de référentiel local nous permet d'avoir une visualisation intéréssante et informative des régions où il persiste une structure des fibres qui démontre le potentiel de contraction d'un coeur. Notre approche pourrait être un moyen prometteur de comprendre le processus de réorgnanisation des fibres du coeur après un infarctus. Elle pourrait également révéler
\end{abstract}


une nouvelle structure géométrique et sa relation à de malheureusement fréquents effets secondaires: tachycardie, arrhythmie et autres anormalités liées. 


\section{TABLE OF CONTENTS}

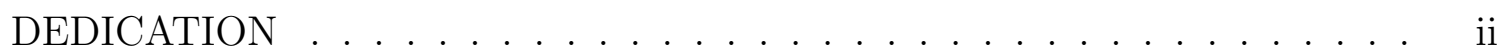

ACKNOWLEDGEMENTS .................... iii

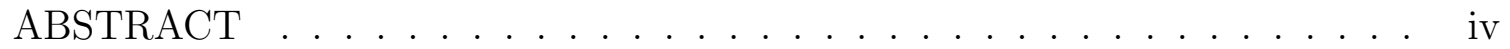

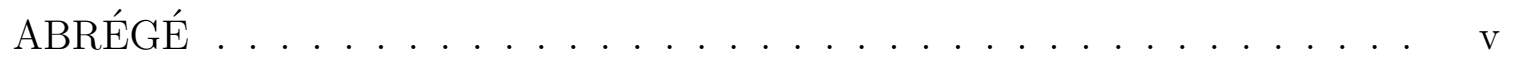

LIST OF TABLES . . . . . . . . . . . . . . . . .

LIST OF FIGURES . . . . . . . . . . . . . . . . . . . xi

1 Introduction . . . . . . . . . . . . . . . . . . . . . . 1

1.1 Objectives and Organization of this Thesis . . . . . . . . 3

1.2 Publications arising from the work in this thesis . . . . . . . 4

2 Literature Review and Experimental Setup . . . . . . . . . . . . . 6

2.1 Literature Review . . . . . . . . . . . . . . . . . 6

2.1 .1 Anatomy of the Heart . . . . . . . . . . . . . 6

2.1.2 Cardiac Diffusion MRI . . . . . . . . . . . . . . . 6

2.1.3 Diffusion Tensor Imaging . . . . . . . . . . . . . . . . . 11

2.1.4 Heart fiber geometry . . . . . . . . . . . . . . 15

2.1.5 Infarcts and their impact on heart fiber geometry . . . . 23

3 Methodology . . . . . . . . . . . . . . . . . 27

$3.1 \quad$ Experimental Setup . . . . . . . . . . . . . . . . . . 27

3.1.1 Data acquisition of pig infarcted and healthy hearts . . . 27

3.1 .2 Source of input data . . . . . . . . . . . . . . . . . . 29

3.1 .3 Quality of our data . . . . . . . . . . . . . . . 30

3.1.4 Usage of MedInria software to read the DICOMs . . . . . . 31

3.2 Rician noise smoothing . . . . . . . . . . . . . . . . . 32

3.3 Getting fiber directions from the diffusion tensor matrix . . . . . . 32 
3.4 Modeling fiber geometry using connection forms . . . . . . . . . 33

3.5 Cartan Frame Fitting and Error Analysis . . . . . . . . . . . . . . 36

4 Results and Discussion . . . . . . . . . . . . . . . . 37

4.1 Quantitative Results . . . . . . . . . . . . . . 37

4.1.1 Histograms of connection forms . . . . . . . . . . 39

4.2 Qualitative Results .................... 41

4.2.1 Importance of Rician filtering . . . . . . . . . . . . . . . 41

4.2.2 Tractography and Fitting . . . . . . . . . . . . . . 41

4.2.3 Error of fit vs ADC and FA . . . . . . . . . . . . . 46

4.2 .4 Discussion .................... 47

5 Conclusion . . . . . . . . . . . . . . . . . . 49

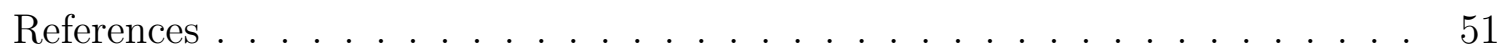




\section{ABBREVIATIONS}

\begin{tabular}{c|c} 
ADC & Apparent Diffusion Coefficient \\
BZ & Border Zone \\
CR & Cardiac Reconstruction \\
CT & Computed Tomography \\
FA & Fractional Anisotropy \\
GHM & Generalized Helicoid Model \\
LAD & Left Anterior Descending \\
LV & Left Ventricule \\
MRI & Magnetic Resonance Imaging \\
RV & Right Ventricule
\end{tabular}


$3-1$ Description of our available datasets . . . . . . . . . . . 30

4-1 Dice and modified coefficients for infarcted hearts . . . . . . . . . . 39

4-2 Total helix angle using our connection form parameters . . . . . . . 41 
2-1 Long axis view of the heart anatomy, adapted from [23] . . . . . . . 7

2-2 Magnetic behavior of the hydrogen nucleus . . . . . . . . . . . . 8

2-3 Torque effect on the magnetic moment of the proton $\mathbf{M}$ when applying an extra magnetic field $\mathbf{B}_{1} \ldots \ldots . \ldots 9$

2-4 Relaxation of the magnetic spin after the magnetic pulse $\mathbf{B}_{\mathbf{1}}$ stops . . 10

2-5 Pulse Gradient Spin Echo (PGSE) sequence . . . . . . . . . . . . . 12

2-6 Examples of possible local frame fields in a world coordinate system $(x, y, z) \ldots \ldots \ldots \ldots \ldots$

2-7 Example of a fiber neighborhood of 27: the center fiber (dark blue) is where we will have a first guess for the values of the connection forms $c_{i j k}$ and then will run an energy minimization algorithm to come up with parameters that give the closest orientation for the neighbors that we can see in the ground truth data here . . . . . . 20

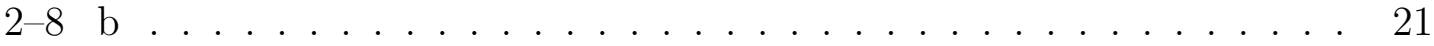

2-9 The helix angle varies smoothly from one tangent plane to the other from the epicardium to the endocardium . . . . . . . . . . . . . 22

3-1 Histology of a transverse slice of an infarcted heart analyzed in [26]. The healthy muscle tissue is represented in red and the scar tissue is stained in blue by a Masson Trichrome stain. . . . . . . . . . . . 29

3-2 $c_{123}(\mathrm{rad} / \mathrm{mm})$ with range of values in porcine hearts . . . . . . . . . 34

3-3 Error of fit, giving the absolute angle difference (in degrees) between our estimation from the connection forms and the ground truth . . 35 
4-1 Histograms of error of fit and connection form parameters for pig \# 6. See text for discussion . . . . . . . . . . . . . . . . . 38

$4-2$ View of the helix angle of heart fibers for pig $4 \ldots \ldots$. . . . . . . . 42

4-3 Tractography of heart fibers for pig $4 \ldots \ldots$. . . . . . . . . . . 42

4-4 Pig 2: Error of fit computed using our framework compared to ADC and FA map . . . . . . . . . . . . . . . . . . . . . . . . 44

$4-5 \quad \operatorname{Pig} 4 \ldots \ldots \ldots \ldots \ldots \ldots$

$4-6 \quad \operatorname{Pig} 5 \ldots \ldots \ldots \ldots \ldots 4 \ldots \ldots \ldots$

$4-7$ Pig $6 \ldots \ldots \ldots \ldots$

$4-8$ Pig $7 \ldots \ldots \ldots \ldots$

$4-9 \quad \operatorname{Pig} 25 \quad \ldots \ldots \ldots \ldots \ldots$ 


\section{CHAPTER 1 Introduction}

The technological improvement of noninvasive medical imaging techniques in the past couple of decades has allowed us to get a better understanding of the heart's structure [34]. This is also aided by the increasing amount of imaging data that can be collected more and more easily and with better definition as time goes by.

As the imaging techniques get better [33], their analysis begins to play a major role in our understanding of the causes and effects of heart disease. Cardiovascular diseases are still the leading global cause of death [22] since 1900 (except for the year 1918) and the knowledge gained by cardiac image analysis has to be used in a clever way to drive them down.

To this day, the most common imaging modalities used by clinicians to get images of the heart are Magnetic Resonance Imaging (MRI), Computed Tomography (CT), and echocardiography. Thus far little work has been carried out in reconstructing models of heart wall fibers and their geometry. The image analysis tools described in $[25,24,26]$ try to come up with a solution to the problem of modeling heart wall fibers from diffusion MR images. This latter problem is important since this solution gives information on the heart wall mechanical function [14].

The core organization of these fibers can be altered by various common pathologies, including rheumatic heart disease or inflammatory heart disease or dilated, 
hypertrophic, restrictive cardiomyopathies, all of which can lead to a deficient contraction of heart wall muscle $[16,4]$.

Numerous former histological studies have shown how fibers in a healthy heart are smoothly wrapped around it and the variation of the helix angle (a measure of fiber orientation) is a common descriptor of local fiber geometry [10]. Computationally aided visualization of the disorganization that can occur in the heart structure could be helpful for modern treatments that feature tissue engineering methods to restore the contractile properties of the heart $[7,17,18,35,44]$, or in more extreme cases where they proceed with reconstruction, or even ablation of infarcted regions $[2,9,15,31]$.

In general the most common means to assessing the impact of a heart disease is via echocardiography, CT and MRI. CT is a good tool to analyze patients with coronary artery diseases as this tool is efficient in revealing different layers in a slice of a body and therefore highlighting foreign objects which for instance could obstruct an artery. Echocardiography, the most often used method to analyze heart disease, can provide numerous useful sources of information about heart shape and size, the flow of input and output blood, but as presently used in the clinic it does not provide information on tissue organization and fiber structure. Although research groups focus on getting fiber orientation from ultrasound, this is still a work in progress and is not yet used in clinical practices.

The imaging of patient specific models of heart wall fibers has become popular using Diffusion MRI methods and has allowed for the investigation of cardiac myofibers and laminar architecture $[29,12,30]$. Despite this enthusiasm, most work 
so far has focused on healthy hearts. Although some work has been done on fiber modeling $[24,32]$ there are very few methods that provide subject specific differential geometric signatures of fiber orientation.

\subsection{Objectives and Organization of this Thesis}

The objective of this thesis is to demonstrate the potential of Maurer-Cartan connection forms to both characterize healthy tissue and indicate regions affected by infarcts via an analysis of their error of fit. This analysis can reveal regions of healthy tissue but also can indicate partially viable tissue in the infarct zone, which is an information that is not directly revealed by ADC or FA scalar measures.

In Chapter 2 we provide a literature review where we overview the imaging methods used and what kind of information we can obtain from these technologies. Then we review current knowledge of heart fiber geometry and demonstrate that Maurer-Cartan connection forms show promise for describing local fiber orientations. We then describe how infarction can scar a region of the heart, which will be the first source of major modification in the heart wall organization, which then remodels itself to lead to a unique geometrical organization of the fibers in the infarct region. Finally the experimental setup will give an overview of the data we were able to test on and how it was obtained, as well as histopathology in some cases that can help us recover the actual heart wall fiber organization.

In Chapter 3, we start from our knowledge of Maurer-Cartan connection forms and use this model to get the most accurate description of the fibers of the heart [24] that we can get. Then we explain how our fitting method works to get those parameters from the data and how our error of fit measurements can indicate healthy 
regions with a coherent fiber orientation throughout the heart wall or confirm the presence of scar tissue and collagen that is observed on the ADC or FA maps.

In Chapter 4, our results are demonstrated and explained with illustrations. There we analyze the extra information that our fitting method and error of fit

measurements provide. We compare the results we obtain with our approach to the ADC and FA scalar maps that are usually referred to, in order to be sure that we get at least similar information about the infarct. Furthermore, the error of fit measurement can show more precisely areas with a high deficiency of coherence in fiber orientation and patches of fibers that still seem to be properly organized to help with the contractile function of the heart.

We conclude by arguing that our approach shows promise while more work could be done in this direction to collect more quantitative results. This would allow our frame fitting method to provide more useful information that is otherwise hard to obtain in a non invasive approach.

\subsection{Publications arising from the work in this thesis}

The preliminary results obtained from the work in this thesis are in the following publications:

- Cartan Frame Analysis of Hearts with Infarcts, poster presented at the 2016 edition of IMNO (Imaging Network Ontario) in Toronto by Damien Goblot, Mihaela Pop and Kaleem Siddiqi.

- Cartan Frame Analysis of Hearts with Infarcts, article to appear in the 2016 volume of LNCS (Lecture Notes on Computer Science), on STACOM 2016. 
The main contributor to the above publications was Damien Goblot. The co-authors helped with the writing, experimental design and interpretation of the results. 


\section{CHAPTER 2}

\section{Literature Review and Experimental Setup}

\subsection{Literature Review}

\subsubsection{Anatomy of the Heart}

Let's introduce the anatomy of the heart as this thesis will focus on the local organization of cardiac muscle fibers. These fibers are bundled together to form the heart wall. The heart is a muscular organ in the shape of a half ellipsoid. The bulk of the heart wall muscle consists of the myocardium, which is bounded by the epicardium, its outer wall, and the endocardium, its inner wall.

The heart chambers we are most interested in are the Left Ventricle (LV) and the Right Ventricle (RV). The RV and LV are significantly different in their structure, and the LV is a much more substantive muscle than the RV since it needs to pump blood to distant organs in the body. The cardiac ventricular muscles are made

of elongated cylindrical cells (cardiomyocytes). The organization of those cells is optimal for efficient pumping to maximize the ejection fraction.

\subsubsection{Cardiac Diffusion MRI}

Magnetic Resonance Imaging (MRI) is the imaging method that was used to acquire the data on which our results are based. It is non-invasive and is adapted to the analysis of soft-tissues [11]. We will explain briefly how it works and what information it gives us. 


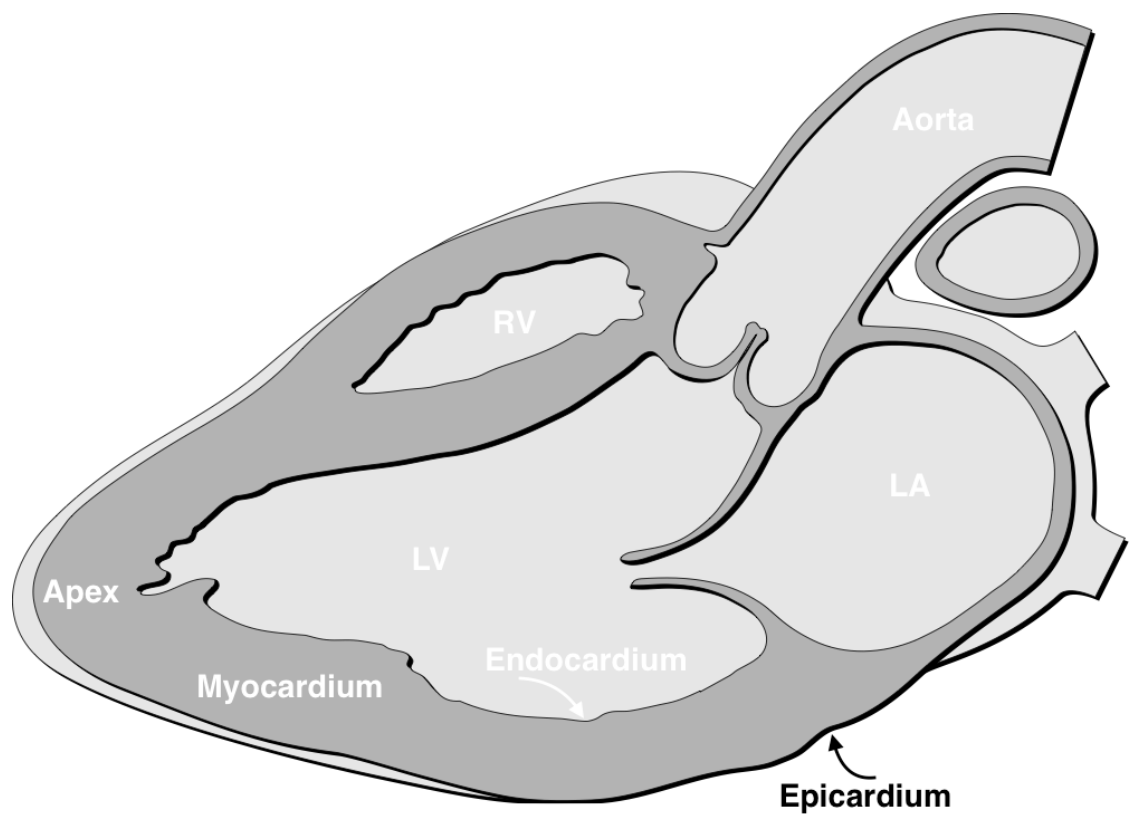

Figure 2-1: Long axis view of the heart anatomy, adapted from [23]

\section{Magnetic Moment of Nuclei}

Magnetic Resonance acquisitions rely on the physical properties of the body, and more specifically the most abundant nucleus which is hydrogen $(\mathrm{H})$. Indeed water molecules $\left(\mathrm{H}_{2} \mathrm{O}\right.$, therefore 2 nuclei of hydrogen in each molecule) represent more than $90 \%$ of the entire body composition. This hydrogen nucleus $\mathrm{H}$ consists of 1 proton of positive charge. The proton spins around itself and therefore creates a magnetic moment which makes the nucleus behave like a magnet to the outside world.

Applying an external magnetic field $\mathbf{B}_{\mathbf{0}}$ leads to a precession around the direction of $\mathbf{B}_{\mathbf{0}}$ of the nuclear magnetic moment of the nucleus. Its magnetic moment will have a characteristic Larmor frequency $\omega_{0}$. All the magnetic moments of the nuclei in a specific region will average out to a net magnetization vector $\mathbf{M}_{\mathbf{0}}$ aligned with $\mathbf{B}_{\mathbf{0}}$. 


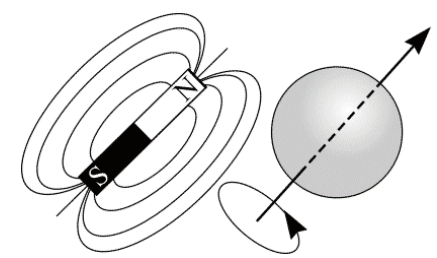

(a) The hydrogen proton behaves like a magnetic dipole - Adapted from $[20]$

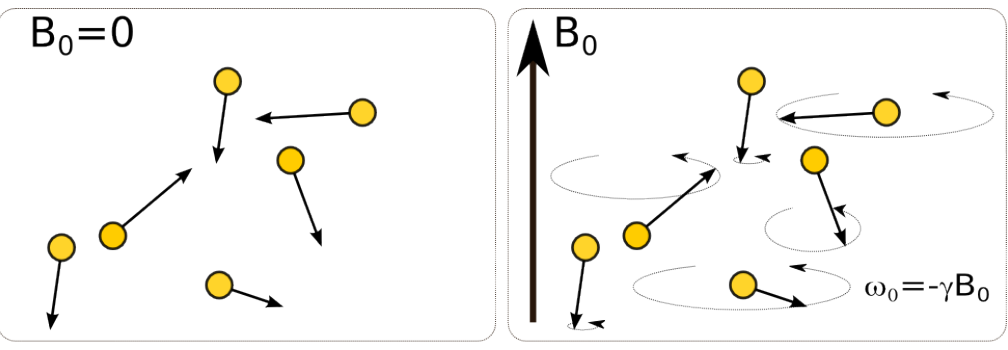

(b) The Larmor precession when an exterior magnetic field is present

Figure 2-2: Magnetic behavior of the hydrogen nucleus

However, it is important to note that all protons will have a different phase and that the precession is asynchronous between all protons, as shown in $2-2 \mathrm{~b}$.

Then the idea is to apply a secondary and much less intense magnetic field $\mathbf{B}_{\mathbf{1}}$, with || $\mathbf{B}_{\mathbf{1}}\left\|\simeq 10^{-6}|| \mathbf{B}_{\mathbf{0}}\right\|$ in a direction in the plane orthogonal to the direction of $\mathbf{B}_{\mathbf{0}}$, rotating around the direction of $\mathbf{B}_{\mathbf{0}}$ with the Larmor frequency $\omega_{0}$ to bring the nuclei to resonance and out of their equilibrium, ie into an excited state, as shown in Fig. 2-3. A torque will indeed be applied to the magnetic moment of the nucleus, coming from the Lorentz force: $\tau=\mathbf{M} \times \mathbf{B}_{\mathbf{1}}$ where $\mathbf{M}$ is the magnetic moment of the nucleus. This can be achieved by transmitting radio frequency (RF) pulses. Then the motion of all nucleii will be synchronized and will be in phase.

Once the impulse stops, the protons will return to their non-excited states and in that process will emit another magnetic signal (weak compared to $\mathbf{B}_{\mathbf{0}}$ ) that will be analyzed, since it is specific to each proton. This information will be used to understand the distribution of the orientations of the magnetic moments in the environment. 


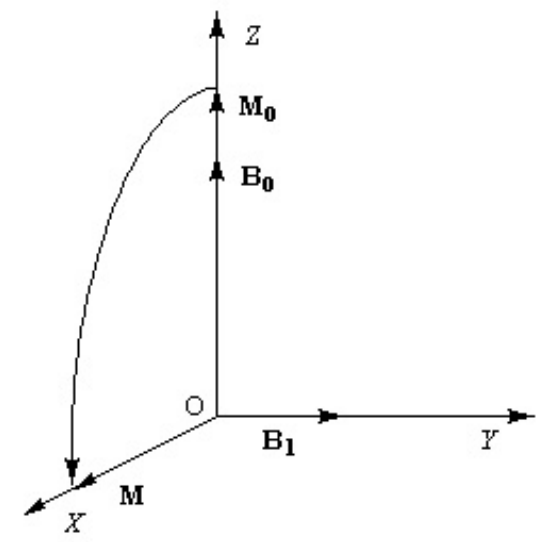

Figure 2-3: Torque effect on the magnetic moment of the proton $\mathbf{M}$ when applying an extra magnetic field $\mathbf{B}_{\mathbf{1}}$

\section{Using Magnetic Resonance for Imaging}

Once a nucleus is in its excited state, its relaxation as illustrated in Fig. 2-4 and the outcome magnetization vector in the $(x, y)$ plane $\mathbf{M}_{\mathbf{x}, \mathbf{y}}$ induces a current recorded by a $\mathrm{RF}$ receiving coil.

This is the signal that is measured in a MR experiment and will be used to acquire the data.

For acquisitions in $2 \mathrm{D}$ or $3 \mathrm{D}$, gradients are applied to the RF pulse (so that the excitation of molecules is characteristic to a location. As a result, the receiving coil can differentiate the localization of the signal more easily depending on this modulation. Typically in 2D frequency and phase encoding is used for the 2 degrees of freedom. In 3D slice selection is accomplished via a linear magnetic gradient, so that the main exterior magnetic field $\mathbf{B}_{\mathbf{0}}(z)$ is different for each slice and leads to different Larmor values for each value of $z$. 


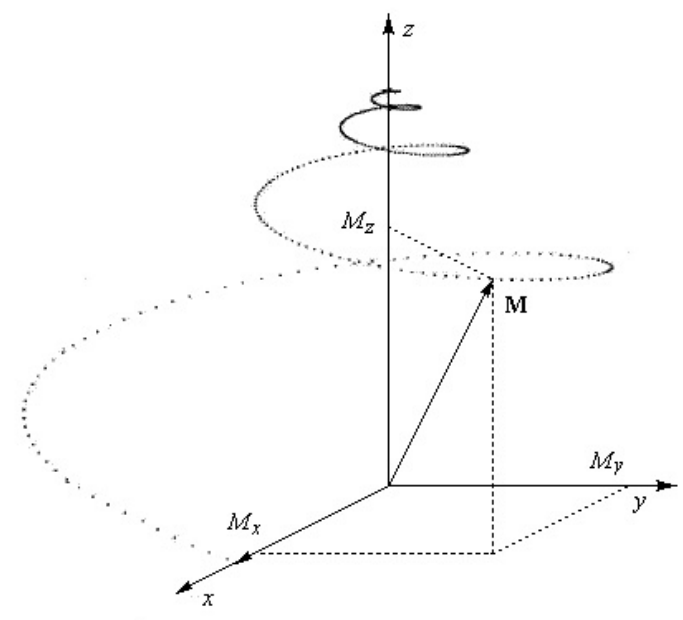

Figure 2-4: Relaxation of the magnetic spin after the magnetic pulse $\mathbf{B}_{\mathbf{1}}$ stops

In our case all MRI are acquired ex-vivo, which means that the acquisition process is more simple as the tissue is perfectly still and several acquisitions can be performed without having to worry about the tissue's motion. In the presence of subject motion more elaborate methods like FLASH imaging have been able to reduce the acquisition time.

Standard MRI is based mainly on the specific relaxation properties of water molecules, and this allows the physician to get images that represent efficiently the different types of tissues depending on their concentration in water molecules - highest in water and fat specifically. Diffusion MRI focuses on the tissue organization by giving out local characteristics of molecular diffusion. This time 2 pulses are applied and the motion of water molecules between these 2 sequences will be characterized. 


\subsubsection{Diffusion Tensor Imaging}

Magnetic resonance diffusion tensor imaging (DTI) is a method based on MRI and the diffusion mechanism of water molecules to assess the direction of fibers from the anisotropic diffusion of these molecules in an oriented or elongated structure.

\section{Physical diffusion property of molecules}

In general the DTI signal is based on the proton of the hydrogen nucleus present in water molecules.

In a non-restrictive volume, the motion of molecules is Brownian and has a Gaussian distribution at equilibrium that is linked to the temperature of the environment and other properties. This Gaussian distribution will be affected by the presence of physical barriers that will decrease the diffusivity. Molecular motion will in fact be hindered in the direction perpendicular to the obstacles. When barriers are present the diffusion will change from isotropic to anisotropic. If we look at a free water molecule, its diffusion probability distribution function would have the shape of a sphere whereas with boundaries in the medium the diffusion of the same water molecule would be more of an ellipsoid with principal direction orthogonal to the boundaries.

\section{Diffusion weighted imaging}

Two short and intense successive pulsed field gradients (PFGs) are applied to the region of interest. The first makes protons have a precession phase proportional to their position along the direction of the gradient. Then a second one is applied with the exact opposite direction so that molecules that didn't move will have a null precession phase whereas those that moved will have a precession phase proportional 

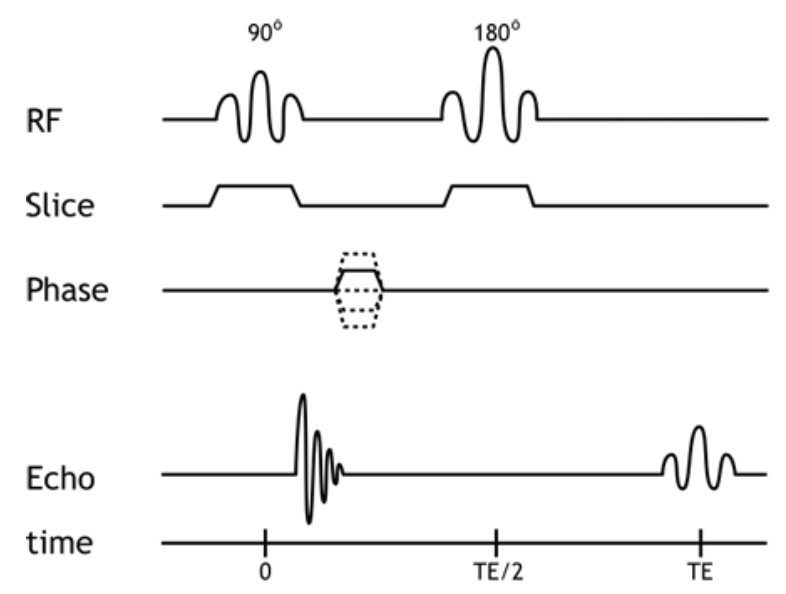

Figure 2-5: Pulse Gradient Spin Echo (PGSE) sequence

to their motion in the direction of the PFG. Here we refer single water molecules but in reality we are considering a distribution of water molecules. For unbounded water molecules the distribution will be centered around a null motion whereas water molecules bounded by a rectangular container for instance will have a motion distribution centered around the direction of the longest vertex.

The acquisition sequence, also called a Pulse Gradient Spin Echo (PGSE) sequence, can be described as follows: [1]

1. A first PFG is applied in a direction orthogonal to the main magnetic field $\mathbf{B}_{\mathbf{0}}$ for $t \in[0, \delta]$.

This will have the effect of flipping the nuclear spins in the direction of the impulse.

2. Once the impulse is over, the magnetic moment of the nuclei will start dephasing as they will start rotating around the direction of $\mathbf{B}_{\mathbf{0}}$, each of them with 
their specific frequency related to the strength of the magnetic field at their specific location.

3. After a diffusion time $t=\Delta$, another PFG is applied but this time with a direction opposite to the first impulse for $t \in[\Delta, \Delta+\delta]$, which will refocus the spin phases that have not moved, and partially refocus those that have moved.

4. Finally at the echo time $t=T E$, the diffusion signal is received by the scanner coil with a loss proportional to the displacement of water molecules during this process. It is the information given by the displacement that is related to the micro structure of the studied environment.

The equation of Nuclear Magnetic Resonance (NMR) was given by Stejskal and Tanner in 1965: it quantifies the decrease in signal intensity and relates this to the apparent diffusion coefficient $\mathbf{D}$.

Let $\mathbf{G}(t)=\left(H\left(t-t_{1}\right)-H\left(t-\left(t_{1}+\delta\right)\right)\right) \mathbf{u}+\left(H\left(t-t_{2}\right)-H\left(t-\left(t_{2}+\delta\right)\right)\right) \mathbf{v}$

where $\mathbf{u}, \mathbf{v}$ are unit vectors in the direction of the applied gradients, and $H(t)$ the Heaviside function at a given time $t$. This function is in fact the mathematical description of the PGSE sequence. Then we get the following Stejskal and Tanner equation $[36]$ :

$$
\frac{S(T E)}{S_{0}}=\exp \left(-\gamma^{2} G^{2} \delta^{2}\left(\Delta-\frac{\delta}{3}\right) \mathbf{G}^{T} \mathbf{D G}\right)=\exp (-b \times \mathbf{D})
$$

where:

- $\gamma$ : gyromagnetic ratio, constant specific to each molecule

- $G$ : PFG strength

- $\delta$ : duration of the pulse 
- $\Delta$ : time between 2 pulses

- D: apparent diffusion coefficient

- $b$ factor suggested by LeBihan [19]: $b=\gamma^{2} G^{2} \delta^{2}\left(\Delta-\frac{\delta}{3}\right)\|\mathbf{G}\|^{2}$

\section{To get a diffusion tensor matrix}

Diffusion anisotropy can be described mathematically by a 3x3 diffusion tensor:

$$
\mathbb{D}=\left(\begin{array}{ccc}
D_{x x} & D_{x y} & D_{x z} \\
D_{y x} & D_{y y} & D_{y z} \\
D_{z x} & D_{z y} & D_{z z}
\end{array}\right)
$$

with $D_{i j}$ the apparent diffusion coefficient (ADC) in direction $(i, j)$. In an environment dominated by water molecules, principles of thermodynamics dictate that $\mathbb{D}$ is symmetric, which means that $D_{i j}=D_{j i}$.

In conclusion we need at least 6 independent gradient directions to determine all 6 degrees of freedom in this matrix. In practice 7 directions are used to get more accurate results, along with a non-weighted measurement for normalization.

The values that we analyze most frequently are ADC and fractional anisotropy (FA). The ADC is an interesting measure as it can show difference in average diffusion values from one region to another - it was particularly adopted to assess the severity of injury in adult stroke patients. It has been regarded as an insufficient source of information recently as it is extremely sensitive to tissue microstructure. As for the $\mathrm{FA}$, it is a very interesting measurement as its value is determined by the environment in which molecules evolve. It is indeed designed to pick up locations with preferential diffusion. The FA value will be high when one eigen value of the diffusion is much 
larger than the others.

$$
\begin{gathered}
A D C=\frac{\operatorname{tr} \mathbb{D}}{3}=\frac{\lambda_{1}+\lambda_{2}+\lambda_{3}}{3} \\
F A=\sqrt{\frac{\left(\lambda_{1}-\lambda_{2}\right)^{2}+\left(\lambda_{2}-\lambda_{3}\right)^{2}+\left(\lambda_{3}-\lambda_{1}\right)^{2}}{\lambda_{1}^{2}+\lambda_{2}^{2}+\lambda_{3}^{2}}}
\end{gathered}
$$

\subsubsection{Heart fiber geometry}

From the 1970s, papers have shown how myofibers in the left ventricle (LV) are aligned along helical curves [32]. This alignment has proved to be optimal for its mechanical function. Heart fibers stay locally packed together and almost parallel to each other, although they are bundled into a special surface: a generalized helicoid [5]. This is a minimal surface that maintains this property through the whole beat cycle. The bundle of myofibers in generalized helicoids appears to be a characteristic feature through the heart wall $[24,32]$.

\section{The Generalized Helicoid Model (GHM)}

We will introduce a local frame field $\mathbf{f}_{1}, \mathbf{f}_{2}, \mathbf{f}_{3}: \mathbb{R}^{3} \rightarrow \mathbb{R}^{3}$ that will allow us to describe more easily the GHM. In this description, we follow the same local frame field definition as chosen in previous publications [24, 32]:

- $\mathbf{f}_{1}$ is the local orientation of the fiber

- $\mathbf{f}_{3}$ is the local heart wall normal

- $\mathrm{f}_{2}=\frac{\mathrm{f}_{3} \times \mathrm{f}_{1}}{\left\|\mathrm{f}_{3} \times \mathrm{f}_{1}\right\|}$ : to get a right-handed orthogonal coordinate frame field

In the GHM model the fiber orientation is expressed at each point $(x, y, z) \in \mathbb{R}^{3}$ by the function:

$$
\theta(x, y, z)=\arctan \left(\frac{K_{T} x+K_{N} y}{1+K_{N} x-K_{T} y}\right)+K_{B} z
$$




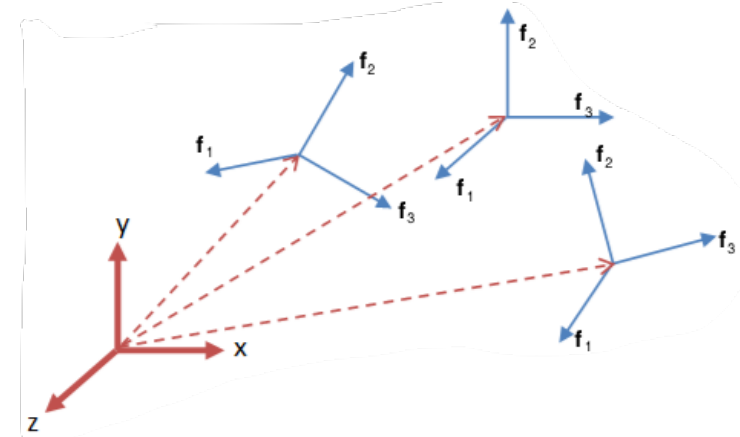

Figure 2-6: Examples of possible local frame fields in a world coordinate system $(x, y, z)$

where $\theta$ is the angle $\angle(\vec{x}, \vec{y})$ and $\vec{x}$ is the local orientation of the fiber and $\vec{y}$ the second axis defining the plane normal to the local heart wall.

- $K_{T}(\mathrm{rad} / \mathrm{mm})$ causes bending in the tangent direction to the fiber

- $K_{N}(\mathrm{rad} / \mathrm{mm})$ makes fibers fan out, away from each other, or towards each other, depending on its sign, in the $\mathbf{f}_{2}$ direction

- $K_{B}(\mathrm{rad} / \mathrm{mm})$ copies rotated versions of the fiber in planes parallel to the original $(x, y)$ plane, i.e. in the direction orthogonal to the heart wall

In general those values describe the amount of change in fiber orientation when going in each of the direction: tangential, orthogonal and out of plane.

Analyses of these parameters on different species have shown on the entire heart volume [32] that the GHM fits with small values of $K_{T}$ and $K_{N}$ and high values of $K_{B}$. This validates the helicoidal model approach and the characteristic of the helix angle in heart myofiber structure.

We now move on from an model based description (GHM) using a fixed reference frame to measure parameters to another approach that is model free. The method 
of moving frames we will introduce in the next section allows us to have a reference frame that can be redefined for each fiber we are focusing on. Whereas in the GHM model, the variation of connection form parameters are coupled such that there are only 3 degrees of freedom $\left(K_{T}, K_{N}\right.$ and $\left.K_{B}\right)$ once the frame is fixed, in the model free approach all 9 connection form parameters can vary.

\section{The Maurer-Cartan form}

The Maurer-Cartan form operator has proved useful in measuring the differential structure of a manifold, and the theory of moving frame has shown good results in the modeling of the heart fiber geometry [24]. The main idea is to apply the theory of moving frames but reverse it: tuning the Maurer-Cartan connection forms can enable us to generate manifolds based on assumptions on the structure that represent in the best way possible the actual heart fiber structure. This approach can be executed by using different fitting methods.

Theory of moving frames. We utilize the framework described in [8] to describe the geometry of fiber orientation in the heart wall via rotations of a frame field that is fit to the diffusion MRI data.

Let a point $\mathbf{x}=\sum_{i} x_{i} \mathbf{e}_{\mathbf{i}} \in \mathbb{R}^{3}$ be expressed in terms of $\left(\mathbf{e}_{\mathbf{1}}, \mathbf{e}_{\mathbf{2}}, \mathbf{e}_{\mathbf{3}}\right)$, the natural basis for $\mathbb{R}^{3}$.

We define a right-handed orthonormal frame field $\mathbf{f}_{1}, \mathbf{f}_{2}, \mathbf{f}_{3}: \mathbb{R}^{3} \rightarrow \mathbb{R}^{3}$.

Each frame axis can be expressed by the rigid rotation $f_{i}=\sum_{i} a_{i, j} \mathbf{e}_{j}$, where $\mathbf{A}=$ $a_{i, j} \in \mathbb{R}^{3 \times 3}$ is a differentiable attitude matrix such that $\mathbf{A}^{-1}=\mathbf{A}^{T}$. 
Treating $\mathbf{f}_{i}$ and $\mathbf{e}_{j}$ as symbols, we can write:

$$
\left[\begin{array}{l}
\mathbf{f}_{1} \\
\mathbf{f}_{2} \\
\mathbf{f}_{3}
\end{array}\right]=\mathrm{A} \times\left[\begin{array}{l}
\mathbf{e}_{1} \\
\mathbf{e}_{2} \\
\mathbf{e}_{3}
\end{array}\right]
$$

Since each $\mathbf{e}_{i}$ is constant, the differential geometry of the frame field is completely characterized by $\mathbf{A}$. Taking the exterior derivative on both sides, we have:

$$
\begin{aligned}
\partial\left[\begin{array}{l}
\mathbf{f}_{1} \\
\mathbf{f}_{2} \\
\mathbf{f}_{3}
\end{array}\right]=(\partial \mathbf{A}) \mathbf{A}^{-1}\left[\begin{array}{l}
\mathbf{f}_{1} \\
\mathbf{f}_{2} \\
\mathbf{f}_{3}
\end{array}\right] \\
=\mathbf{C}\left[\begin{array}{l}
\mathbf{f}_{1} \\
\mathbf{f}_{2} \\
\mathbf{f}_{3}
\end{array}\right]
\end{aligned}
$$

where $\partial$ denotes the exterior derivative, and $\mathbf{C}=(\partial \mathbf{A}) \mathbf{A}^{-1}=\left(c_{i, j}\right) \in \mathbb{R}^{3 \times 3}$ is the Maurer-Cartan matrix of connection forms $\left(c_{i, j}\right)$.

Writing $\mathbf{f}_{i}$ as symbols, Eq. 2.7 is to be understood as $\partial \mathbf{f}_{i}=\sum_{j} c_{i, j} \mathbf{f}_{j}$.

The Maurer-Cartan matrix is skew symmetric with zeros as diagonal entries so there are at most 3 independent, non-zero 1-forms: $c_{12}, c_{13}$, and $c_{23}$.

$$
\mathbf{C}=\left(\begin{array}{ccc}
0 & c_{12} & c_{13} \\
-c_{12} & 0 & c_{23} \\
-c_{13} & -c_{23} & 0
\end{array}\right)
$$


1-forms operate on tangent vectors through contraction, written as $\partial \omega\langle\boldsymbol{v}\rangle \in \mathbb{R}$ for a general 1-form $\partial \omega=\sum_{i} \omega_{i} \mathbf{e}_{\mathbf{i}}$ and tangent vector $\boldsymbol{v} \in \mathbb{R}^{3}$, which yields:

$$
\begin{aligned}
\partial \omega\langle\boldsymbol{v}\rangle & =\sum_{i} \omega_{i} \partial \mathbf{e}_{\mathbf{i}}\left\langle\sum_{j} v_{j} \mathbf{e}_{\mathbf{j}}\right\rangle \\
& =\sum_{i} \omega_{i} v_{i}
\end{aligned}
$$

since

$$
\partial \mathbf{e}_{i}\left\langle\mathbf{e}_{j}\right\rangle=\delta_{i, j}
$$

where $\delta_{i, j}$ is the Kronecker delta. It turns out that the space of linear models for smoothly varying frame fields is parametrized by the 1 -forms $c_{i, j}$. Since only 3 unique non-zero combinations of $c_{i, j}$ are possible, there are in total 9 connections $c_{i j k}$ that fully characterize the local frame field geometry.

Tuning connection form parameters by energy minimization. Finding out the correct values for the connection form parameters $c_{i j k}(\mathbf{x}), \forall \mathbf{x} \in \mathbb{R}^{3}$ is done by energy minimization within a neighborhood $\Omega$, as we can see in Fig. 2-7. This approach makes sense as the GHM is still dependent on the location in the heart, indeed the rate of turning will not be the same close to the apex for instance or where the heart wall is thinner.

Therefore the optimal parameters are defined as follows:

$$
\begin{aligned}
c_{i, j, k}^{*}(\mathbf{x}) & =\operatorname{argmin}_{c_{i j k}} \frac{1}{\Omega} \sum_{\mathbf{v} \in \Omega} \sum_{i=1}^{3} \epsilon_{i}(\mathbf{x}+\mathbf{v}) \\
\epsilon_{i}(\mathbf{x}+\mathbf{v}) & =\arccos \left(\mathbf{f}_{i}(\mathbf{x}+\mathbf{v}) \cdot \tilde{\mathbf{f}}_{i}(\mathbf{x}+\mathbf{v})\right)
\end{aligned}
$$




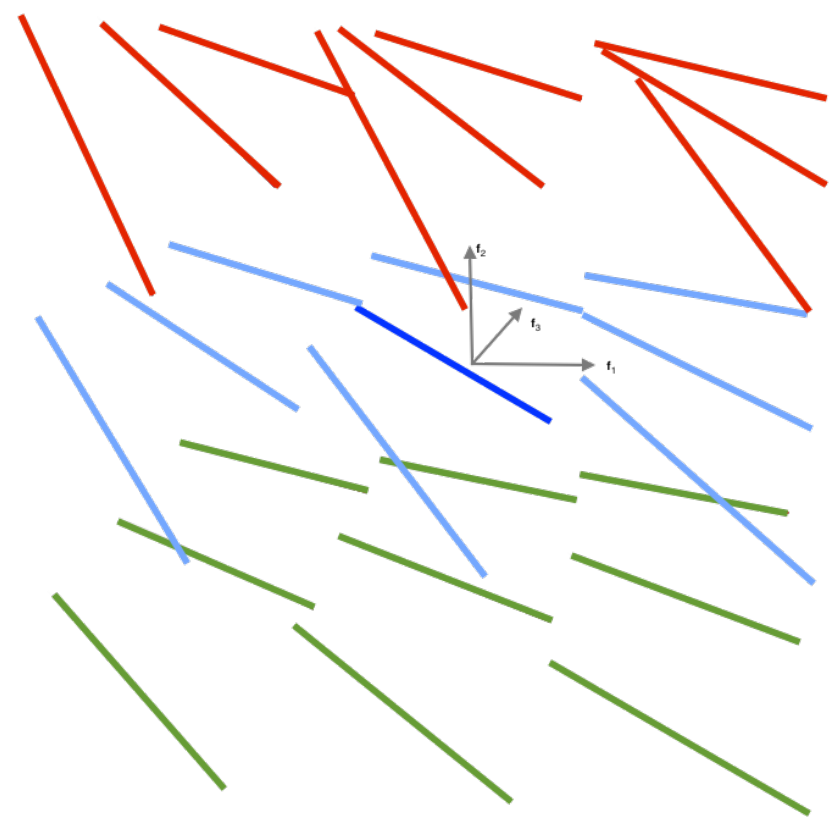

Figure 2-7: Example of a fiber neighborhood of 27: the center fiber (dark blue) is where we will have a first guess for the values of the connection forms $c_{i j k}$ and then will run an energy minimization algorithm to come up with parameters that give the closest orientation for the neighbors that we can see in the ground truth data here

where:

- $\epsilon_{i}$ is the error function for each axis

- $\mathbf{f}_{i}$ is the actual frame orientation at this location

- $\tilde{\mathbf{f}}_{i}$ is the current approximation of the frame orientation

The optimization strategy can be one of the standard well-known algorithms for these specific tasks: either Nelder-Mead [40] or BOBYQA [28]. 


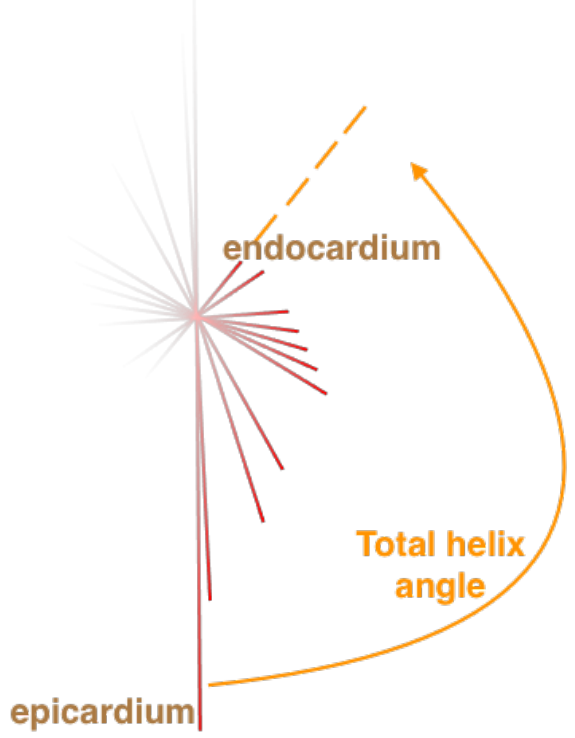

Figure 2-8: Visualization of the helix angle with a transmural point of view

\section{Combining both for a good description of heart fiber geometry}

The heart is made of elongated muscle cells called cardiomyocytes that are organized within a collagen matrix. The macroscopic shape is one of a truncated ellipsoid, while on a microscopic scale myocytes are stacked and form myofibers [37].

Work on histology has shown how cardiac myofibers wrap around the ventricles in helical curves [6]. The ubiquitous analysis of myofiber geometry states out that the helix angle - $\theta$ or $c_{123}$ in previous sections, or the angle $\angle\left(\overrightarrow{\mathbf{f}_{1}^{i}}, \overrightarrow{\mathbf{f}_{2}^{i+1}}\right)$ where $i$ is a tangent plane to the heart wall at this location - varies smoothly in Fig. 2-9 with a total change in Fig. 2-8 of around $120^{\circ}$ for mammals.

Applying this method to the heart can be done by choosing $\mathbf{f}_{i}$ as described previously in the GHM model. $\mathbf{f}_{1}$ is easy to set from the DTI data, while $\mathbf{f}_{3}$ is 


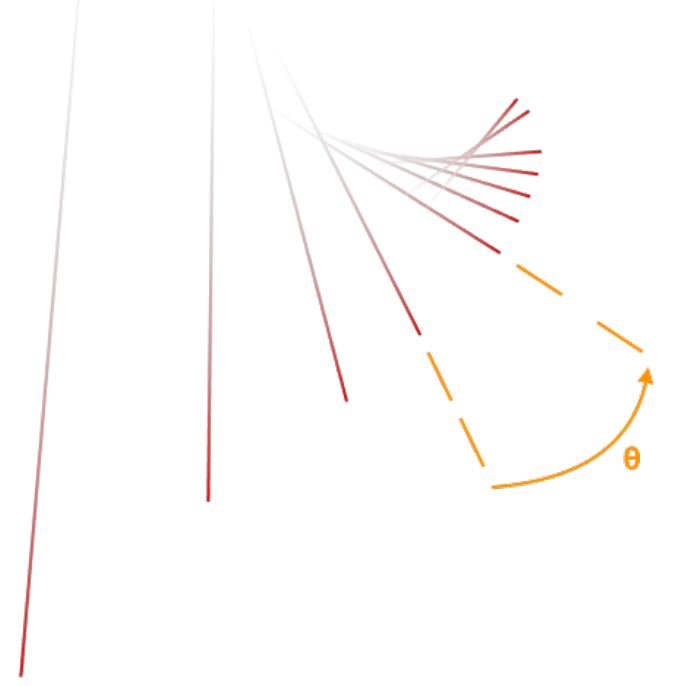

Figure 2-9: The helix angle varies smoothly from one tangent plane to the other from the epicardium to the endocardium

determined locally by an approximation of the transmural direction at each location using a mask of the studied heart and a euclidian distance computed between the epicardium (outermost layer of the heart wall) and endocardium (innermost layer) of the heart.

The Maurer-Cartan connection form approach is a good one to describe the cardiac frame field, where the 3 main geometrical features are exhibited by parameters of this model:

- $c_{123}$ for the helix angle, or trans-mural penetration of the heart wall

- $c_{131}$ quantifies the short-axis (in plane for a given slice) curvature of the heart wall

- $c_{232}$ represents the long-axis (from one slice to another) curvature 
As a reminder, in general, these coefficients $c_{i j k}$ express the rate of turn of the frame vector $\mathbf{f}_{i}$ towards $\mathbf{f}_{j}$ when $\mathbf{x}$ moves in the direction $\mathbf{f}_{k}$.

In previous sections we reviewed MRI and DMRI as they are the technologies involved in the acquisition of our data. Then a review of the GHM and the Maurer-Cartan forms helped us introduce the tools we rely on for the geometric characterization of fiber orientation. In the following section we provide the reader a brief review of infarcts and their effects, as it is the main application area of this thesis. This subject is particularly interesting as not much is yet known about how fibers are remodeled in the presence of infarcts.

\subsubsection{Infarcts and their impact on heart fiber geometry}

Studies have been trying to determine the consequences of myocardial infarctions in small (rat [41]) hearts and larger (porcine [43, 27]) hearts. In these studies it was shown that acute infarction led to a decrease in regional wall thickness and an increased radius of curvature, mostly in the border zone (BZ) - which is the zone close to the dense scar region but with a more heterogeneous nature due to the persistence of surviving blood vessels that continue to supply oxygen to isolated cells.

\section{Myocardial remodeling after infarct}

After an infarct occurs, a remodeling happens in the heart, which means that

the normally existing structure goes through a rearrangement process as a result of the infarct [38]. Cardiac remodeling involves a number of phases including the adaptation of myocytes and collagen to new geometrical conditions, fibrosis due to increased collagen, and finally the death of cells as a result of fibrosis. 
One of the first consequences of cardiac reconstruction is a decrease of the wall thickness, which leads immediately to an increased volume of the Left Ventricle (LV). Another direct effect is cardiac heart hypertrophy, with a multiplication of non-muscle cells (fibroblasts and endothelial cells) in the place of former muscle myocytes. A study on rats [21] showed that the hypertrophy lead to a $25 \%$ increase of the heart mass 13 weeks after infarct.

Myocardial fibrosis is one of the major signs of malignancy in the cardiac reconstruction process, which can lead to death by cardiac failure or severe arrhythmias.

\section{Effects of wall stress on tissue composition and geometry}

One of the issues in the study of the consequences of an infarct on a mammal heart is the significant cost that it involves. As imaging is expensive, only a few were carried out, which makes the analysis even more specific to the few examples available for study [13]. Several experiments have been carried out and the following chronological evolution of cardiac fiber geometry has emerged:

- Local infarct expansion 1 week after the initial infarct, characterized by a stretch in a plane tangent to the epicardium and wall thinning.

- Scar shrinkage 3 weeks after infarction. The cardiac reconstruction, even without reestablishing a contractile function as efficient as before the infarct, works around the infarct to compensate the work overload on the scar region.

- Alignment of myocardial collagen fibers with the direction of the greatest applied stress, which takes place during infarct healing 


\section{Characteristics of collagen in infarcted hearts and arrangement of the surviving fibers}

Studies [21] have shown that after the occurrence of an infarct the whole heart mass increases by an average of $25 \%$. The infarcts were affecting the LV, and the difference in the LV mass could involve up to an $58 \%$ increase.

A major impact of an infarct on the heart is on the collagen concentration that goes up significantly both in the scar tissue, the Border Zone which is specifically rich in fibrosis and in more distant regions. The concentration of collagen can double in the most affected regions of the heart. In general and throughout the whole heart the collagen concentration increases dramatically and is combined with more crosslinking of these cells.

Studies on human hearts [8] have shown that arrhythmias and tachycardia can be caused by abnormal geometrical arrangement of the myocardial fibers.

Transmembrane potentials were taken and these showed little difference between patients with ventricular tachycardia in a chronic phase of myocardial infarction and normal patients. This clearly indicates that the surviving myocardial fibers within an infarct can come back close to normal after a CR process. Surviving bundles in the subendocardial layers have also been observed, as well as intramurally and subepicardially. These bundles of surviving myocytes showed an anisotropy similar to the papillary muscle of the heart, and therefore a similar coupling resistance, which makes sense as the activation signal propagates itself like a zigzag through the infarcted region of the heart.

After a transmural myocardial infarction, the outermost epicardial muscle survives and its structure contributes greatly in the occurrence of the re-entry ventricular 
arrhythmia. These surviving muscle cells in the BZ of the epicardium are like a thin sheet over the infarct. The connections with other intramural muscle is not present very often, and this means that the impulses are only 2-dimensional as they cannot come from the bottom part of the heart, which prevents an appropriate circular movement and can lead to tachycardia [39]. 


\section{CHAPTER 3 Methodology}

Our processing pipeline uses a combination of open source software for research purposes (MedInria) and developed in our lab for our very specific needs of reproducible experiments [25]. As explained in the previous section the first step is to use MedInria to read the DICOMs and get the tensor matrix presented in Section 2.1.3 at every voxel, as well as the FA and ADC in Matlab file format.

In this chapter we will explain how Cartan frames and the approximations of the 1-forms are an appropriate fit for the data that we have. This will be illustrated by some examples from our dataset. The detailed experimental results on the pig hearts along with the discussion of those results will be presented in Chapter 4 .

\subsection{Experimental Setup}

The data on which our results are based are all porcine hearts that were provided to us by Dr. Mihaela Pop of the Sunnybrooke Research Institute and the Department of Medical Biophysics at the University of Toronto.

\subsubsection{Data acquisition of pig infarcted and healthy hearts}

Each porcine heart studied in this thesis were freshly excised, suspended in a plexiglass phantom filled with fluorinert (to eliminate artifacts) and placed in an MR head coil for ex-vivo imaging. All DW-MR studies were performed on a dedicated 1.5T GE Signa Excite scanner using a custom FSE pulse sequence. They used the following MR parameters [26]: 
- $T E=35 \mathrm{~ms}$

- $T R=700 \mathrm{~ms}$

- echo train length $=2$

- $b=0$ for the unweighted MR images. As a reminder, the $\mathrm{b}$ factor is explained in Section 2.1.3

- $b=500 \mathrm{~s} / \mathrm{mm}^{2}$ for the images where the 7 diffusion gradients were applied

- $256 \times 256 \mathrm{k}$-space

- Field of View FOV $=10-16 \mathrm{~cm}$

- Slice Thickness $=1.2 \mathrm{~mm}$, yielding a sub-millimetric voxel size

From each heart, select samples containing an infarct were cut to align with the short-axis view of the MR images and prepared for histopathology to confirm the collagen deposition in the infarct area.

One of the difficulties that remains is that of finding a good correspondence between the slices from the histology and the ones that were obtained from the MR imaging that occurred before the histopathology was performed. Fig. 3-1 shows a histology image of a slide from an infarcted heart, with intact myocytes in the normal tissue and altered tissue microstructure in the infarcted zone. As depicted by the Masson Trichrome stain, the ischemic border zone (BZ) had collagen fibrils interdigitated between viable myocytes. In the dense scar area, necrotic myocytes were completely replaced by mature fibrosis (the final product of collagen degradation), resulting in the loss of myocardial anisotropy. 


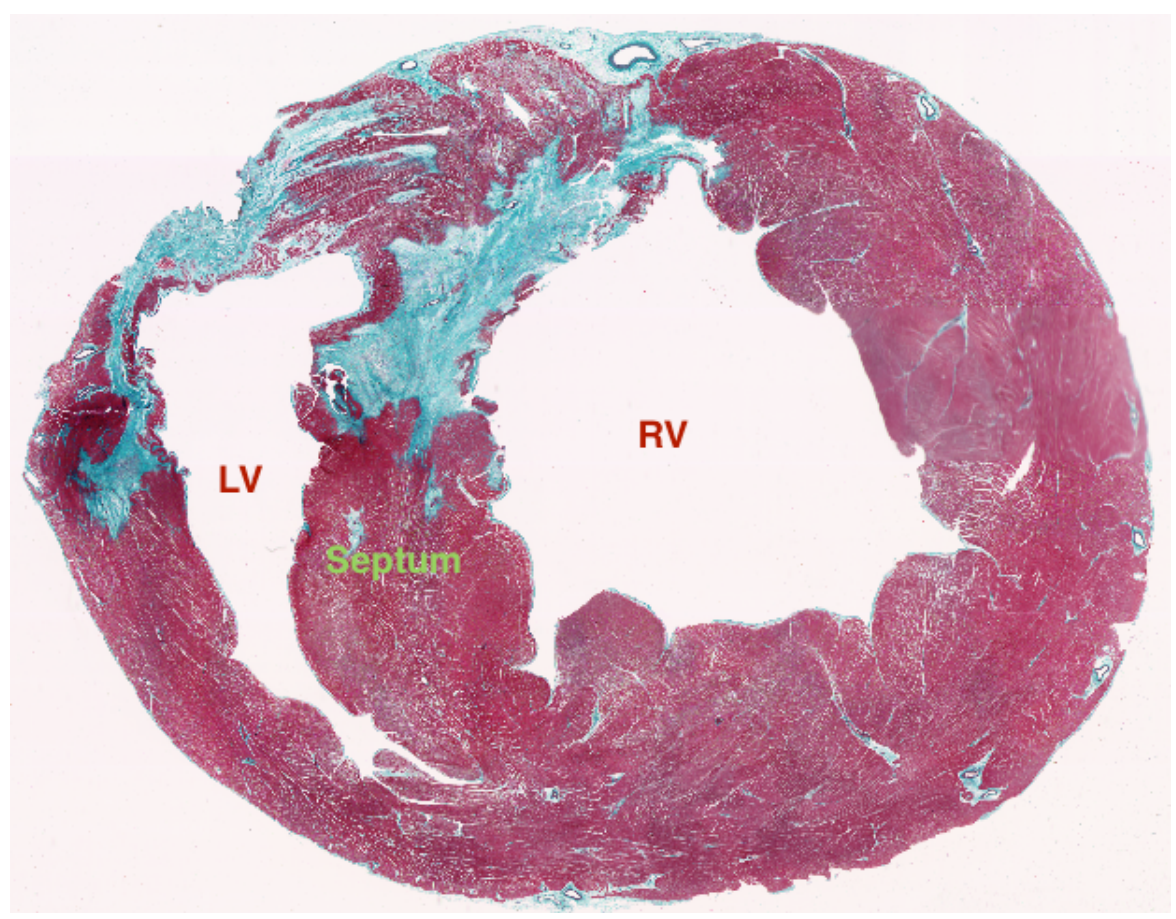

Figure 3-1: Histology of a transverse slice of an infarcted heart analyzed in [26]. The healthy muscle tissue is represented in red and the scar tissue is stained in blue by a Masson Trichrome stain.

\subsubsection{Source of input data}

The data we used [26] are DT-MRI of porcine hearts. Of these 8 hearts had infarcts while 2 were healthy. For every infarcted heart, the imaging process took place 6 weeks after the occurrence of the infarct and histological section was carried out after that for some of the cases. The availability of some histological data allows us to compare our analysis qualitatively with measurements that serve as ground truth. We provide in Table 3-1 an overview of the data we had access to and its usability. 


\begin{tabular}{|c|c|c|}
\hline $\begin{array}{c}\text { Heart } \\
\text { (Related to Acquisition Date) }\end{array}$ & Infarcted or Healthy & Region of infarct \\
\hline 2 & Infarcted & $\begin{array}{l}\text { Left Circumflex artery } \\
\text { (LCX) }\end{array}$ \\
\hline 4 & Infarcted & $\begin{array}{c}\text { Left Anterior Descending } \\
\text { (LAD) }\end{array}$ \\
\hline 5 & Infarcted & LCX \\
\hline 6 & Infarcted & LCX \\
\hline 7 & Infarcted & $\begin{array}{l}\text { Rigth Coronary Artery } \\
\text { (RCA) }\end{array}$ \\
\hline 17 & Infarcted & Bad quality \\
\hline 18 & Infarcted & Bad quality \\
\hline 23 & Infarcted & Unreadable \\
\hline 25 & Healthy & - \\
\hline 28 & Healthy & Too Noisy \\
\hline
\end{tabular}

Table 3-1: Description of our available datasets

\subsubsection{Quality of our data}

For a better and more accurate analysis of our results, we will focus mostly on the best quality data (least noisy) we had access to and fortunately this includes one control heart (\#5) and 5 infarcted hearts. Results for all hearts will be presented in the next Chapter.

Due to the imaging technology used, and to the fact that a fairly low magnetic field strength MRI scanner was used to acquire this data (1.5T), a good amount of noise is present in the raw data. We have taken advantage of knowledge of the type of noise (Rician noise) that is present in MRI-acquired data to apply non-local denoising strategies which are able to better reconstruct the fiber orientation without oversmoothing [3]. The denoising was done by a non local Rician smoothing. The 
probability distribution differs from a Gaussian distribution by a factor of the signal, which makes the mapping non-linear. The two distributions are described as follows:

- Gaussian: $P(x \mid \sigma, \mu)=\frac{1}{\sqrt{2 \pi \sigma^{2}}} \exp ^{-\frac{(x-\mu)^{2}}{2 \sigma^{2}}}$

- Rician: $P(x \mid \sigma, \nu)=\frac{x}{\sigma^{2}} \exp ^{-\frac{\left(x^{2}+\nu^{2}\right)}{2 \sigma^{2}}} I_{0}\left(\frac{x \nu}{2 \sigma^{2}}\right)$

The latter distribution is therefore far from a Gaussian for small Signal-To-Noise (SNR) ratios. The Rician smoothing strategy does not smooth regions where the data is chaotic due to the infarct or due to proximity to the heart wall boundary. A fine tuning of the smoothing strategy allowed us to get good quality datasets without removing relevant information in regions of interest around the infarcts.

\subsubsection{Usage of MedInria software to read the DICOMs}

We took the advantage of the MedInria software to load the DICOM images, pre-process the data via Rician denoising and finally use its Diffusion Tensor Imaging tool to compute the value of the tensor matrix (detailed in Section 2.1.3) at each voxel location in the heart.

From the tensor matrices at every location, we were easily able to compute the fiber orientation at every location, associating it with the direction of the first principal eigenvector. Since the actual direction is arbitrary (modulo $180^{\circ}$ flips) we chose a direction that provided a consistent cylindrical winding around the heart wall, as in [24].

Once we have the fiber orientation at every voxel, we can use our previous work [24] to compute the connection forms and get a sense of the quality of the fitting, as we will describe in the following chapter. 


\subsection{Rician noise smoothing}

We used an established Rician smoothing method to deal with noise in the diffusion images [42]. This is a type of non-local smoothing technique which uses voxel to voxel similarity to guide the smoothing process. In general the parameters for this filtering method must be tuned to prevent oversmoothing, which can happen for instance if the weight is not sufficiently penalized when the similarity is not high enough. This was the very first step of our data processing pipeline.

\subsection{Getting fiber directions from the diffusion tensor matrix}

Once we have our diffusion tensor matrix $\mathbb{D}$, a simple eigenvector and eigenvalue

calculation gives us the eigenvalues $\left(\lambda_{1}, \lambda_{2}, \lambda_{3}\right)$ and the corresponding eigenvectors $\left(\nu_{1}, \nu_{2}, \nu_{3}\right)$.

$$
\begin{aligned}
\mathbb{D} & =\left(\begin{array}{lll}
D_{x x} & D_{x y} & D_{x z} \\
D_{y x} & D_{y y} & D_{y z} \\
D_{z x} & D_{z y} & D_{z z}
\end{array}\right) \\
& =\mathbb{P}^{-1} \tilde{\mathbb{D}} \mathbb{P}
\end{aligned}
$$


where:

$$
\begin{gathered}
\tilde{\mathbb{D}}=\left(\begin{array}{ccc}
\lambda_{1} & 0 & 0 \\
0 & \lambda_{2} & 0 \\
0 & 0 & \lambda_{3}
\end{array}\right) \\
\tilde{\mathbb{D}} \cdot \nu_{1}=\lambda_{1} \nu_{1} \\
\tilde{\mathbb{D}} \cdot \nu_{2}=\lambda_{2} \nu_{2} \\
\tilde{\mathbb{D}} \cdot \nu_{3}=\lambda_{3} \nu_{3}
\end{gathered}
$$

The biggest eigenvalue in absolute value will correspond to the fiber direction and sorting those will give us the eigenvector corresponding to the fiber direction. We can also at this point get the FA and ADC values to double check what MedInria gave us.

All the eigenvectors at every voxel we obtain from this process do not always point in the same direction, and sometimes neighboring voxels will have fibers pointing in opposite direction. To solve this issue, a cylindrical consistency processing step was applied [24]. The idea is to compute from the mask of the heart studied a gradient pointing at each voxel from the inner wall to the outer wall. Once this gradient is obtained, this preprocessing step ensures that all cross products of the eigenvectors and the gradient are of the same sign. If not, then we just change the sign of each component of the eigenvector, flipping it by $180^{\circ}$.

\subsection{Modeling fiber geometry using connection forms}

Connection forms measure the local rotations of the frame axes $\mathbf{f}_{1}, \mathbf{f}_{2}, \mathbf{f}_{3}$. Here we focus on the contraction of the 1 -form $c_{12}$ on the frame axes $\mathbf{f}_{3}$ and compare its 
values in a short axis slice of a pig heart with an infarct - Fig. 3-2a - with those in a short axis slice from a healthy pig heart - Fig. 3-2b.

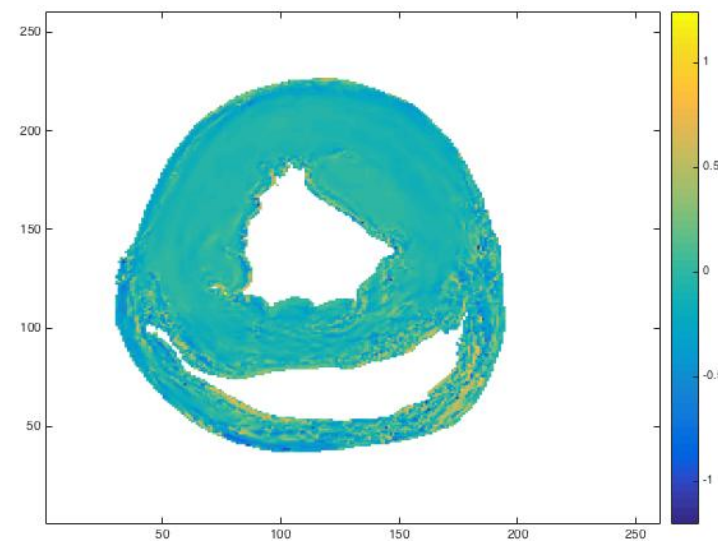

(a) $c_{123}$ (infarcted in the $\mathrm{LAD}$ region). The color scheme varies a lot and contains a lot of yellow which stands for an abnormally high turning of fibers, which means the model could not converge at these locations because of the lack of consistency in the fiber directions

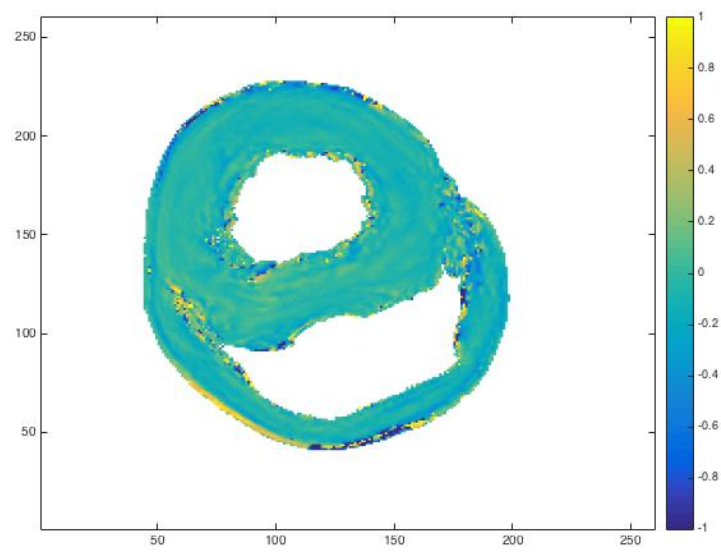

(b) $c_{123}$ (healthy). The colors are smooth and consistent which is characteristic of a smooth turning of fibers throughout the heart

Figure 3-2: $c_{123}(\mathrm{rad} / \mathrm{mm})$ with range of values in porcine hearts

In a qualitative sense, observing the colormap and comparing the smoothness of figures $3-2 \mathrm{a}$ to the control heart in Fig. 3-2b, we can already notice how in the healthy case and in regions remote from the infarct in the infarcted case we have smooth and regular colors which represent a rather constant value of $c_{123}$. On the other hand, in the infarct region (LAD) it is clear that the values vary a lot more and give a strong impression of a lack of coherence. A more quantitative approach will be discussed in Section 4.1.1.

Cartan frame field analysis applies to smoothly rotating frame fields. In the presence of infarcts fiber orientation coherence is lost. The connections then fail to 


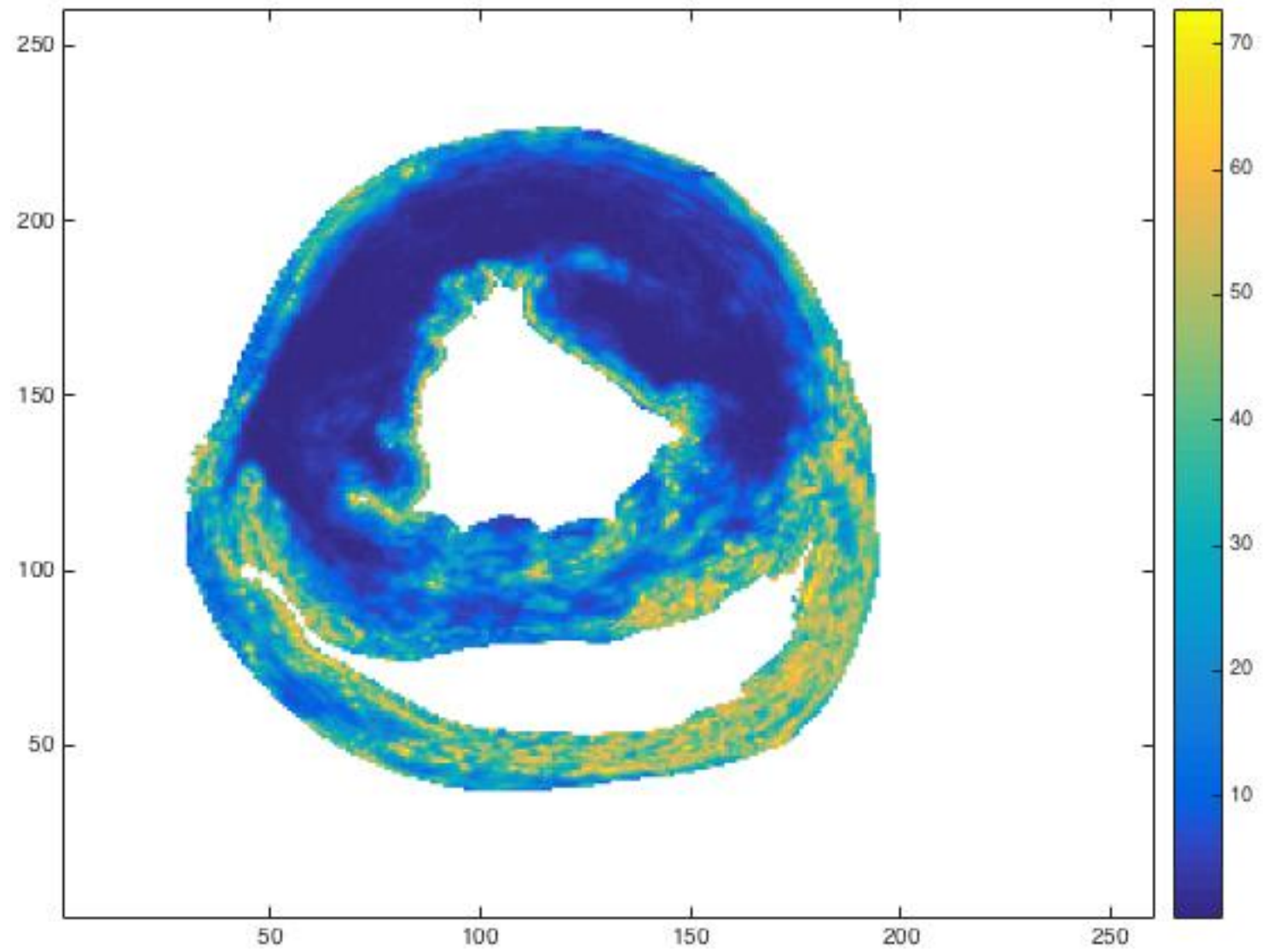

Figure 3-3: Error of fit, giving the absolute angle difference (in degrees) between our estimation from the connection forms and the ground truth 
explain the orientation of fibers in a local neighborhood and fitting errors using this method go up as we can observe in Fig. 3-3. This association of frame field fitting error with fiber incoherence is the key insight behind the developments in this thesis.

\subsection{Cartan Frame Fitting and Error Analysis}

As explained earlier, at each voxel we use an estimate of the fiber orientation given by the orientation of the first principal eigenvector of a diffusion tensor reconstruction for $\mathbf{f}_{1}$. We then estimate the heart wall normal as the gradient of the distance function to the boundary of the myocardium and take the component of the normal that is orthogonal to $\mathbf{f}_{1}$ to be $\mathbf{f}_{3} . \mathbf{f}_{2}$ is then taken to be their cross product. Our numerical implementation of frame field fitting relies on finding the best estimates of the 9 connection forms at each voxel in the sense of explaining the orientations in its neighborhood. Specifically, using Nelder-Mead optimization, we minimize an energy given by the angle between the measured orientation at each neighbor and that given by rotating the frame field by a particular set of connections, explained in Section 2.1.4. Once this method converges the error of fit at a voxel is taken to be the average angular error between fiber orientations in a neighborhood and those given by rotating the frame at that voxel using its connection parameters. 


\section{CHAPTER 4 Results and Discussion}

Now that we have a better understanding of how we use the Cartan frames and approximations of 1 -forms in order to deduce fiber orientation at each location in the heart while computing an error of fit, we will now discuss the results obtained when applying this pipeline to different heart diffusion MRI datasets.

\subsection{Quantitative Results}

The top 3 rows of Figure 4-1a and Figure 4-1b display the distribution of the values of $c_{i j k}$ throughout the whole heart. The last row represents the distribution of the error of fit (in rad/voxel) for each of the 3 local frame directions $\mathbf{f}_{1}, \mathbf{f}_{2}$ and $\mathbf{f}_{3}$.

Given the association between ADC and our error of frame fit, it is natural to compare these measures quantitatively throughout the myocardium. We did so for the 5 infarcted porcine hearts. We analyzed by computing Dice coefficients to describe the overlap, in the following manner:

For the same heart let $\mathrm{A}$ be the set of voxels with $\mathrm{ADC}$ value $>0.6$ and let $\mathrm{B}$ be the set of voxels with error of fit $>15^{\circ}$. Whereas these thresholds were chosen based on empirical conditions, they gave high Dice coefficients across the several porcine

data sets we analyzed. We computed the standard Dice coefficient $\frac{A \cap B}{A \cup B}$ as well as a modified coefficient $\frac{A \cap B}{A}$.

These results demonstrate that typically over $80 \%$ of the locations with increased diffusion also yield a high error of fit using our frame fitting method, due to the loss 

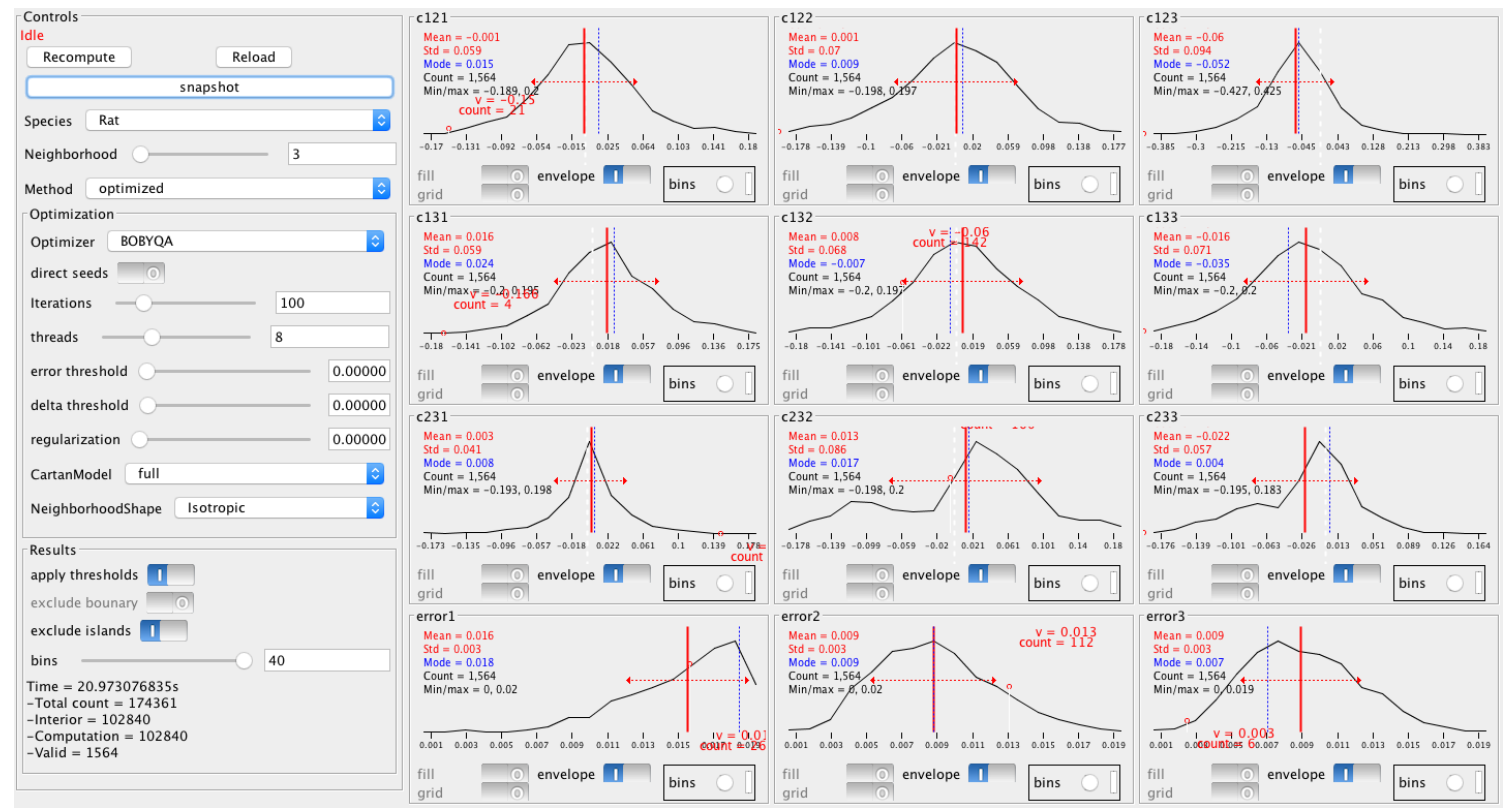

(a) Without any smoothing: the histograms are not sharply peaked and the errors of fit are very high

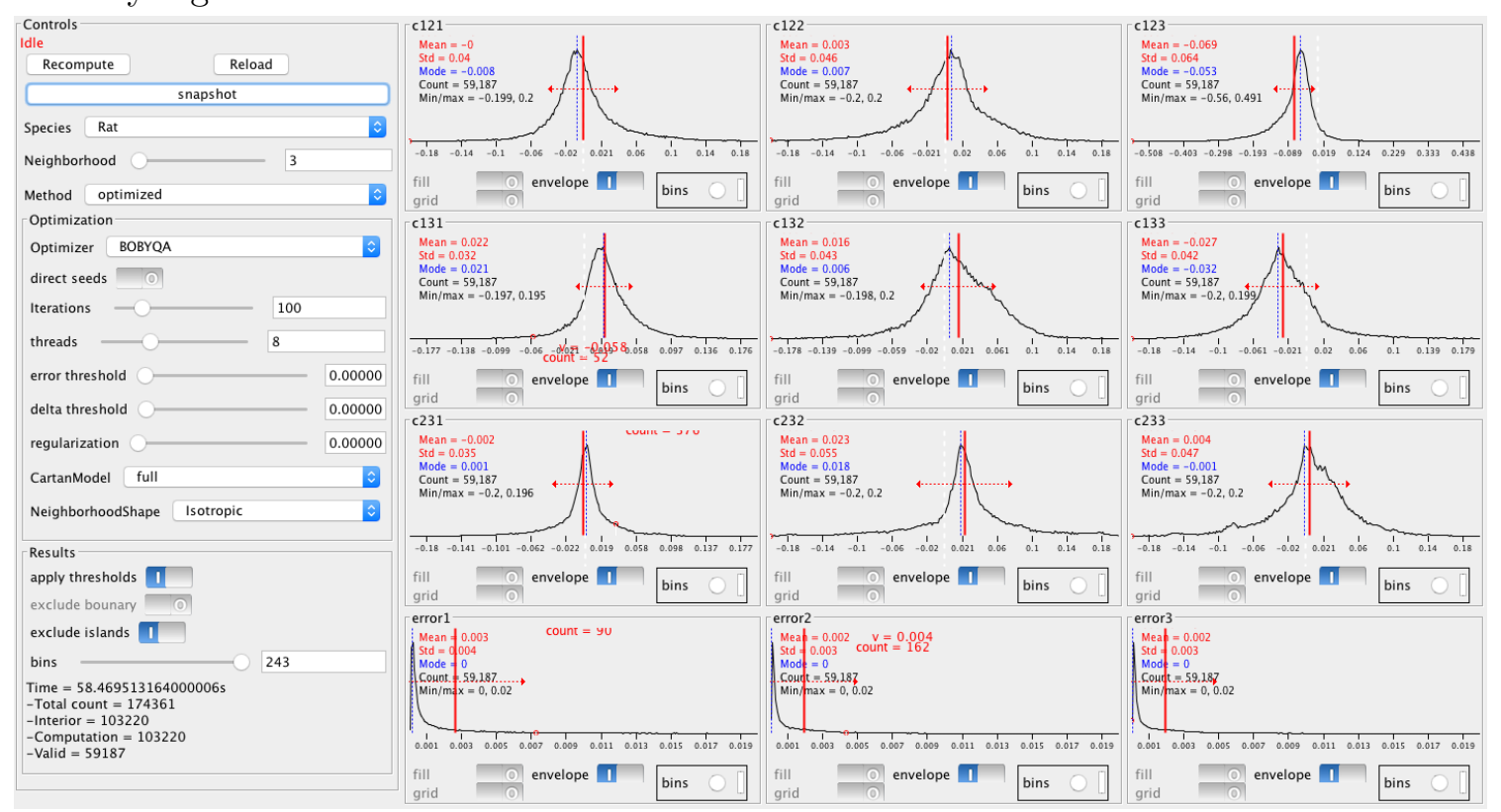

(b) With Rician smoothing: the histograms are more sharply peaked and the errors of fit are much smaller

Figure 4-1: Histograms of error of fit and connection form parameters for pig \# 6 . See text for discussion 


\begin{tabular}{|c|c|c|}
\hline Heart & $\frac{A \cap B}{A \cup B}$ & $\frac{A \cap B}{A}$ \\
\hline 2 & 0.40 & 0.80 \\
\hline 4 & 0.43 & 0.89 \\
\hline 5 & 0.47 & 0.76 \\
\hline 6 & 0.46 & 0.87 \\
\hline 7 & 0.27 & 0.94 \\
\hline
\end{tabular}

Table 4-1: Dice and modified coefficients for infarcted hearts

of geometric coherence of fiber orientations. However there are additional locations where fiber orientations are not smooth, typically at the linings of the heart wall, or near the edges of a collapsing and narrow right ventricle. These are picked up by our error of fit measure but not by the ADC measure, likely because there is no increase in collagen there.

\subsubsection{Histograms of connection forms}

We provide an example of one histogram for pig \# 6 that we computed on the entire heart. This was performed on every dataset. The histogram in Fig. 4-1b gives us information on the distribution of $c_{i j k}, \forall(i, j, k) \in[1,3]^{3}$ and the distribution of the error of fit for each frame axis $\mathbf{f}_{1}, \mathbf{f}_{2}$ and $\mathbf{f}_{3}$. Smoothing in Fig. $4-1 b$ gives us more peaked histograms and far lower error of fit than in Fig. 4-1a. In the way we have set up the local frame field, $c_{123}$ corresponds to turning from outer to inner wall in the sense of the helix angle [24].

In this thesis we will focus on the most commonly used parameter for comparison, the parameter related to the notion of helix angle $c_{123}$. Our tool gives us the mean and the mode (value most represented) of this value through the whole heart. Given the distribution of these values the mode is relevant since it will represent 
mostly fibers in the core of the heart wall but without taking into account (as the mean value does) extreme values that can occur on the boundaries of the heart wall.

The papillary muscles, to name only one anatomical structure within the heart, which are mostly aligned in a top-down direction along the long axis, can have a non-negligible effect on the $c_{123}$ value and they are difficult to remove from our data. Removing them would be useful as they do not play a role in the heart function that we want to study. These muscles are indeed difficult to remove from the data since it is not always clear which layer (in the long axis direction) they start and end in. This is why segmenting them automatically is not obvious. Moreover, thresholding the ADC value to segment tissue with oriented structure does pick up the papillary muscle since it has fibers aligned in a long axis direction.

In Table 4-2 we regroup the results we obtained from all relevant datasets and analyze the total helix angle in degrees that we obtain the following way (where 57.3 is the conversion from $\operatorname{rad}$ to $\left.{ }^{\circ}\right)$ :

$$
\text { orientation from outer to inner wall }=c_{123} \times \# \text { voxels } \times 57.3
$$

The variability is too high to be able to draw conclusions from these values, but they can be helpful to understand at a bigger scale the distribution of the fiber orientations we obtained. This high variability is due to the unability of the algorithm to converge at every voxel, due to the loss of coherence of the fibers in some locations. 


\begin{tabular}{|c|c|c|c|c|c|}
\hline Heart & $\begin{array}{c}\text { Thickness } \\
\text { (\# voxels) }\end{array}$ & $\begin{array}{c}c_{123} \text { mean } \\
\text { rad/voxel }\end{array}$ & $\begin{array}{c}c_{123} \text { mode } \\
\text { rad/voxel }\end{array}$ & $\begin{array}{c}\text { Total helix angle } \\
\text { (using mean) }\end{array}$ & $\begin{array}{c}\text { Helix angle } \\
\text { (using mode) }\end{array}$ \\
\hline 2 & 40 & -0.066 & -0.025 & $-151.2^{\circ}$ & $-57.3^{\circ}$ \\
\hline 4 & 45 & -0.084 & -0.047 & $-216.6^{\circ}$ & $-121.2^{\circ}$ \\
\hline 5 & 30 & -0.048 & -0.020 & $-82.5^{\circ}$ & $-34.38^{\circ}$ \\
\hline 6 & 30 & -0.069 & -0.053 & $-118.6^{\circ}$ & $-91.1^{\circ}$ \\
\hline 7 & 45 & -0.077 & -0.053 & $-198.5^{\circ}$ & $-136.6^{\circ}$ \\
\hline 25 & 35 & -0.072 & -0.034 & $-144.4^{\circ}$ & $-68.2^{\circ}$ \\
\hline
\end{tabular}

Table 4-2: Total helix angle using our connection form parameters

\subsection{Qualitative Results}

\subsubsection{Importance of Rician filtering}

The transverse view in Figure 4-2 helps to show the latter counterclockwise fiber turning. These snapshots are also helpful to visualize the importance of smoothing, as they show clearly that the noise is reduced by a significant amount.

The region shown in the foreground of Figure 4-2a and Figure 4-2b is a region away from the infarct where we can see a smooth turning of fibers. Taking a closer look at these figures show that fibers away from the infarct are indeed not affected and are still organized in a similar way to those in a healthy heart.

\subsubsection{Tractography and Fitting}

Using the fiber orientation that we can get from the computed tensor matrix, we can run tractography on our results. This process gives us an idea of how fibers should be wrapped around the heart and work together in the muscle structure. The way tractography works is fairly simple: the algorithm follows the direction of the fiber and when it reaches a neighboring voxel, it picks up this new direction and propagates further. The infarct region, as explained in [43], is the region with the 

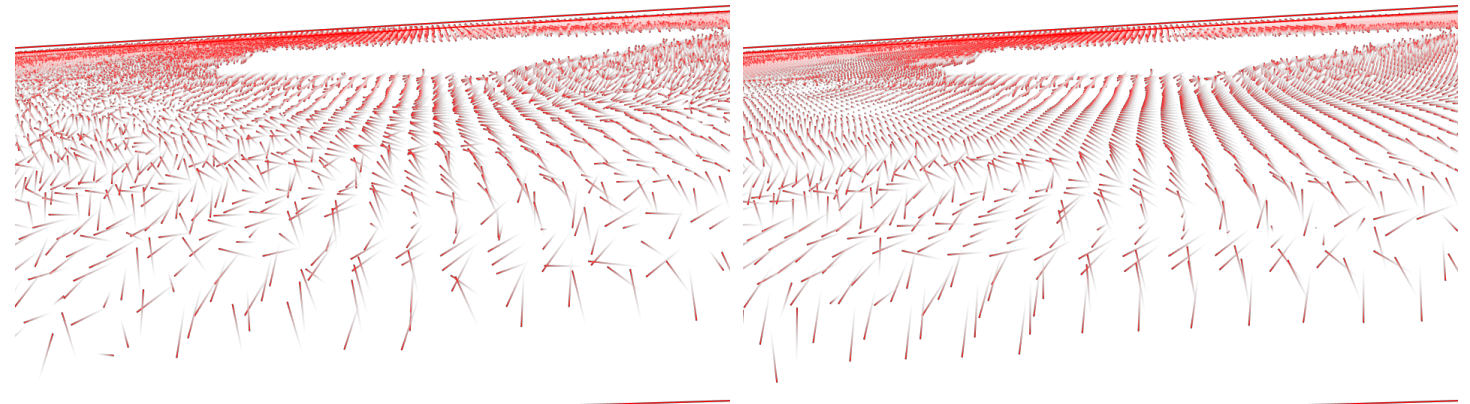

(a) Without any smoothing

(b) With Rician smoothing

Figure 4-2: View of the helix angle of heart fibers for pig 4

least coherence in its fiber directions whereas regions remote from the infarct are not affected in their structure. The tractography provides a more striking visualization of the regions that lack geometrical regularity. Indeed regions not affected by the infarct have their fibers wrapped smoothly along the heart wall in an organized manner.

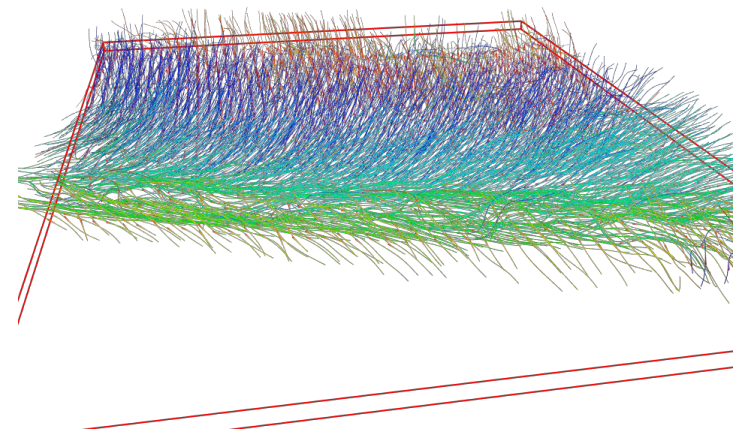

(a) Without any smoothing
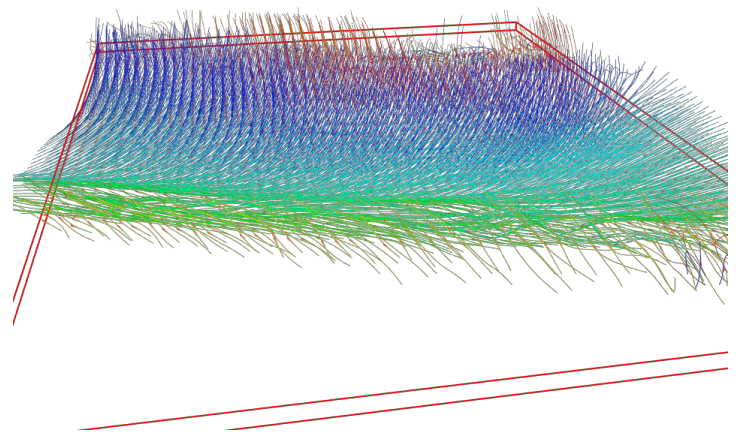

(b) With Rician smoothing

Figure 4-3: Tractography of heart fibers for pig 4 
The histograms provided in Figure 4-1a and Figure 4-1b provide measurements on different subjects:

1. With our dataset Rician smoothing is critical to be able to fit a model on the data. The algorithm was able to successfully apply the fitting method only for 1,564 voxels in the case of the unfiltered data (out of around 90,000). When Rician smoothing was applied, the algorithm converged for 59,187 of these voxels, and therefore gave much more meaningful data.

2. Most fibers have their connection form parameters in the same range, and that is why most datapoints are closely centered around the mode. Previous studies [25] have shown that the variance in values of the first due to intrasubject variability is negligible compared to the one we can witness here. This is another indication that in the case presented here the general fiber structure of the heart was unchanged, although the deviation around the mode accounts for some irregularities in the infarct zone and close to boundaries of the inner and outer heart wall.

3. Most connection form parameters are fairly concentrated around 0, except for $c_{123}$ which accounts for the change in orientation of the fiber direction when moving from outer to inner wall.

The number of voxels that count in our computations is the number of voxels where the algorithm could converge to a value for each of the connection form parameters $\left(c_{i j k}\right)_{(i, j, k) \in \mathbb{R}^{3}}$. When the algorithm could not converge to a stable value after a given number of iterations, it automatically discarded that voxel. 


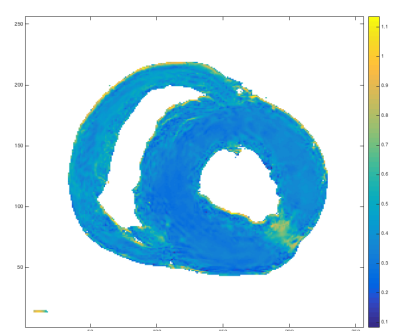

(a) ADC map

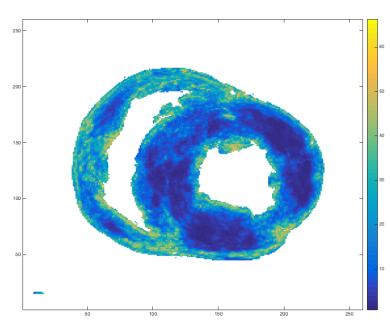

(b) Error of fit

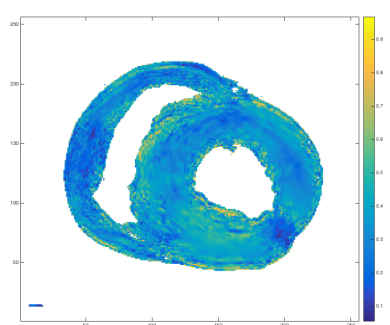

(c) FA map

Figure 4-4: Pig 2: Error of fit computed using our framework compared to ADC and FA map

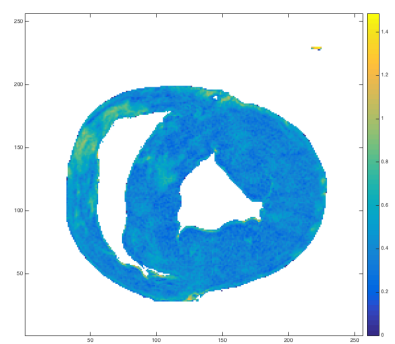

(a) ADC map

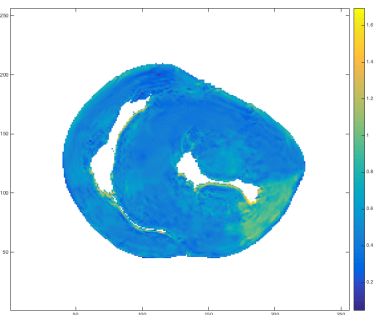

(a) ADC map

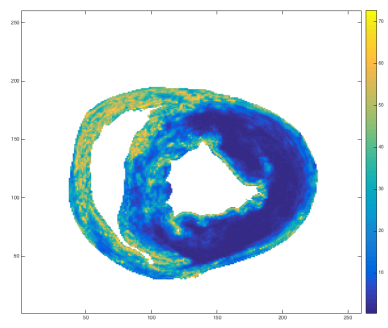

(b) Error of fit

Figure 4-5: Pig 4

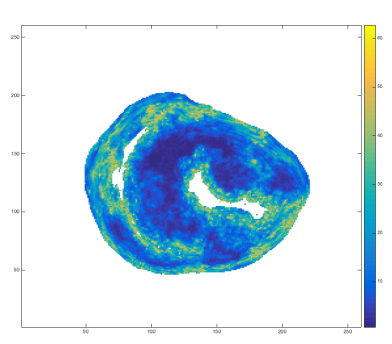

(b) Error of fit

Figure 4-6: Pig 5

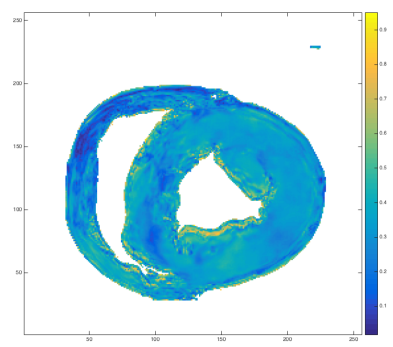

(c) FA map

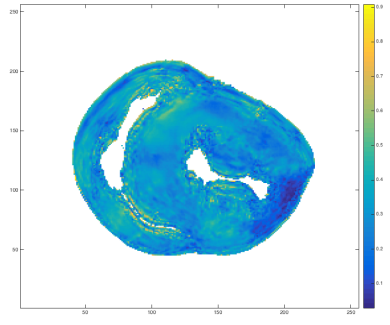

(c) FA map 


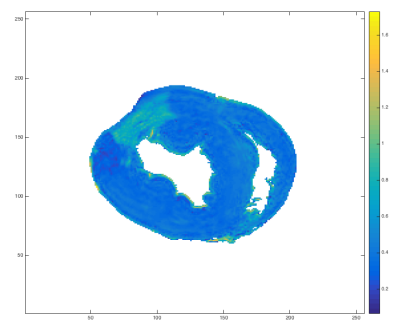

(a) ADC map

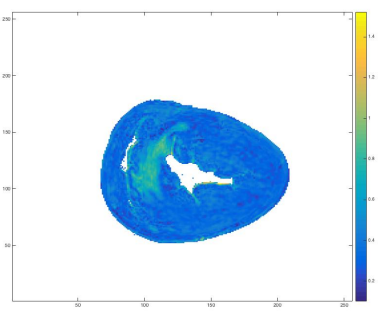

(a) ADC map

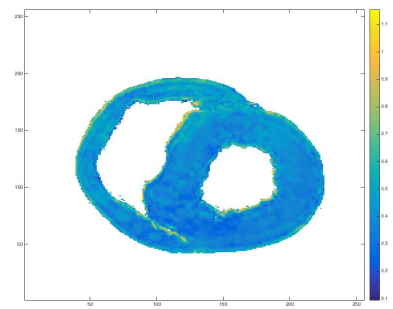

(a) ADC map

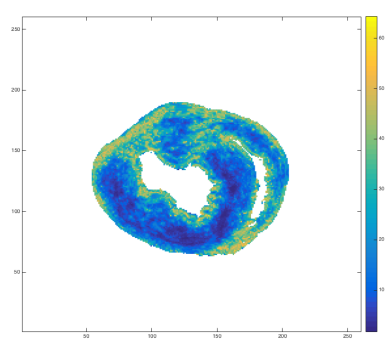

(b) Error of fit

Figure 4-7: Pig 6

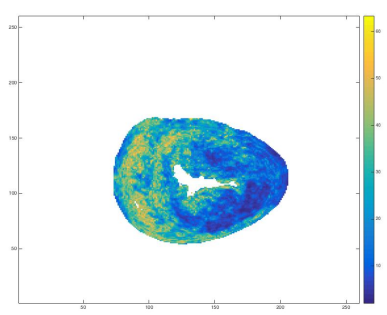

(b) Error of fit

Figure 4-8: Pig 7

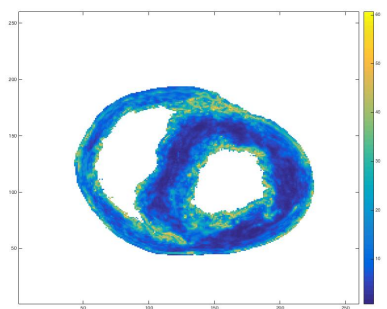

(b) Error of fit

Figure 4-7: Pig 6

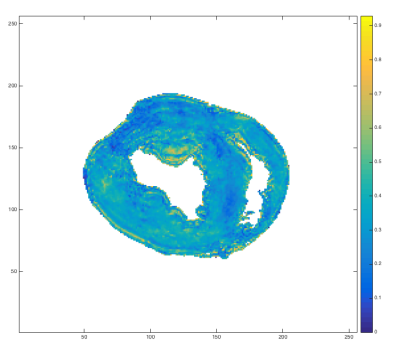

(c) FA map 


\subsubsection{Error of fit vs ADC and FA}

Here we provide a detailed overview of the data that we worked on to get the presented results. It is clear that the error of fit can provide supplementary information compared to what the ADC and FA scalar maps can give us. A high ADC due to increased apparent diffusion in collagen and a low FA due to the lack of coherence of fibers in the infarct region is expected.

If we focus on the first example - Figure 4-4, we can see in the ADC map shown in Figure 4-4a the region of the infarct where the value is the highest (yellow region). The FA map goes accordingly. In that precise region there is no observable location where the ADC would be a little bit lower, whereas the error of fit varies more smoothly and shows regions (close to the inner wall for instance) where the error is lower. This region could be a patch of surviving fibers that are still somewhat organized to help the contractile function of the heart. The advantage of the error of fit is that it could more discriminative as a local measure at a voxel. On the contrary, the FA and ADC maps look more like ink spots that broaden around the infarct region. A direct comparison with histology would be a very important addition to assess the results obtained by the error of fit.

This arrangement of the surviving fibers as described in the literature overviewed in Section 2.1.5 is also clearly visible in the examples displayed in Figure $4-5$ and Figure 4-6.

The example in Figure 4-7 is also very interesting, as it shows, as mentioned in [39], how muscles can be almost completely absent in certain regions. In this case 
it seems that only collagen is present in the infarct area, with few surviving fibers contributing to wrapping the entire ventricle.

Our approach seems to be more specific and precise in the exhibition of surviving fibers in the infarct region and in some cases shows the possible survival of some fibers which could contribute to the contractile function of the heart.

The last provided example, Figure $4-9$, is used as a control healthy heart to make sure of the extent to which we can use our results. We can clearly see in this healthy example that the error of fit is low almost everywhere, except in regions near the heart wall (endothilial and epithilial linings) and the region where the Left Ventricle (LV) and the Right Ventricle (RV) connect to each other. In the septum (between the LV and the RV), there are two different directions of rotation right next to each other, and the direct implementation of our moving frame method is not designed to pick it up. Indeed in this case the neighborhood of a voxel can contain fibers wrapping around the LV and fibers wrapping around the RV. These neighbor fibers have a completely different orientation and are responsible for the high error of fit in this region.

\subsubsection{Discussion}

In all the examples seen in the previous section, locations where the ADC value is high correspond roughly with ones where the error of fit is high. This is to be expected since a high ADC value corresponds to areas with more collagen and less fibrous tissue. The high error of fit is a sign of lack of consistency in the orientation of fibers, which can mean two things. Either the region is less populated with healthy 
and organized fibers, or the region observed does not contain fibers but is comprised of other tissue.

The error of fit shows additional information that could support existence of surviving fibers in the infarct and border zone that can help the contractile function of the heart after the infarct. This knowledge would be very useful as it is a noninvasive way to qualify the capacity of the heart to keep beating and pumping blood to the body despite the occurrence of an infarct. Further experiments could be carried out to support this analysis and show how the efficiency of an infarcted heart is related to the remaining fibers, e.g., blood flow could be measured before sacrificing the hearts for an ex-vivo diffusion MRI scan.

Another very promising research topic would be to analyze the evolution of surviving fibers at different time periods after the occurrence of an infarct. The present study was limited to the datasets available, which were all obtained 6 weeks after the infarct. 


\section{CHAPTER 5 \\ Conclusion}

The study of infarcted pig hearts 6 weeks after infarction has allowed us to compare the more mainstream analytical approach, that is to use ADC and FA maps, to our error of fit analysis using Cartan frames fits.

We have noticed a difference in the results shown by the scalar approaches (FA and ADC) compared to our geometrical approach that tries to understand and explain how the tissue is organized and how fibers are geometrically oriented. As the literature points out, after the occurrence of an infarct surviving fiber bundles can persist in the infarct region and have a role in the contraction of the damaged heart. Geometrical reorganization is interesting to observe as it is the cause to tachycardia. Our method offers a promising non-invasive approach to assess the effects of an infarct. More advanced analytics on the location specific evolution of the connection

form parameters could be promising to understand heart wall remodeling after an infarct.

The limitations in this development are the lack of complete and easy to compare histology slices of the hearts we analyzed. This could be a very useful addition to the conclusions that are drawn purely from an error of fit analysis. Another big limitation was the quality of the DMRI data. In spite of our analysis being noninvasive, we have seen that the quality of the data is an issue and unfortunately Rician smoothing did not alway result in improved results. Rician smoothing also 
changes the measurements at certain locations, so in a strict sense the estimated fiber orientations may not correspond with the ground truth ones. High quality in-vivo DMRI data would be a big help to obtain better results.

Another interesting study could be performed on infarcted hearts at different stages in time. An ideal case would be to have good quality in-vivo diffusion MRI images, in order to understand better the effect of the infarct on heart wall fibers, week by week, and the relationship to certain post infarct symptoms such as tachycardia. 


\section{References}

[1] Haz-Edine Assemlal, David Tschumperlé, Luc Brun, and Kaleem Siddiqi. Recent advances in diffusion mri modeling: Angular and radial reconstruction. Medical image analysis, 15(4):369-396, 2011.

[2] Constantine L Athanasuleas, Gerald D Buckberg, Alfred WH Stanley, William Siler, Vincent Dor, Marisa Di Donato, Lorenzo Menicanti, Sergio Almeida de Oliveira, Friedhelm Beyersdorf, Irving L Kron, et al. Surgical ventricular restoration in the treatment of congestive heart failure due to post-infarction ventricular dilation. Journal of the American College of Cardiology, 44(7):14391445, 2004.

[3] Saurav Basu, P. Thomas Fletcher, and Ross T. Whitaker. Rician noise removal in diffusion tensor mri. In MICCAI, 2006.

[4] Mirza Faisal Beg, Patrick A Helm, Elliot McVeigh, Michael I Miller, and Raimond L Winslow. Computational cardiac anatomy using mri. Magnetic resonance in medicine, 52(5):1167-1174, 2004.

[5] DE Blair and JR Vanstone. A generalization of the helicoid. Minimal submanifolds and geodesics, pages 13-16, 1978.

[6] Gerald D Buckberg. Basic science review: the helix and the heart. The Journal of Thoracic and Cardiovascular Surgery, 124(5):863-883, 2002.

[7] Arnold I Caplan and James E Dennis. Mesenchymal stem cells as trophic mediators. Journal of cellular biochemistry, 98(5):1076-1084, 2006.

[8] Jacques MT de Baker, Ruben Coronel, Sara Tasseron, Arthur AM Wilde, Tobias Opthof, Michiel J Janse, Frans JL van Capelle, Anton E Becker, and George Jambroes. Ventricular tachyrdia in the infarcted, langendorff-perfused human heart: Role of the arrangement of surviving cardiac fibers. Journal of the American College of Cardiology, 15(7):1594-1607, 1990. 
[9] Marisa Di Donato, Michel Sabatier, Vincent Dor, Gian Franco Gensini, Anna Toso, Mauro Maioli, Alfred WH Stanley, Constantine Athanasuleas, and Gerald Buckberg. Effects of the dor procedure on left ventricular dimension and shape and geometric correlates of mitral regurgitation one year after surgery. The Journal of thoracic and cardiovascular surgery, 121(1):91-96, 2001.

[10] Liesbeth Geerts, Peter Bovendeerd, Klaas Nicolay, and Theo Arts. Characterization of the normal cardiac myofiber field in goat measured with mr-diffusion tensor imaging. American Journal of Physiology-Heart and Circulatory Physiology, 283(1):H139-H145, 2002.

[11] K. Nicolaij A.J. Bakermans G.J. Strijkers H.C. van Assen, P.H.M. Bovendeerd. Myocardial structure and function of the healthy rat heart. Master's Thesis, 2008.

[12] Patrick Helm, Mirza Faisal Beg, Michael I Miller, and Raimond L Winslow. Measuring and mapping cardiac fiber and laminar architecture using diffusion tensor mr imaging. Annals of the New York Academy of Sciences, 1047(1):296$307,2005$.

[13] JW Holmes, H Yamashita, LK Waldman, and JW Covell. Scar remodeling and transmural deformation after infarction in the pig. Circulation, 90(1):411-420, 1994.

[14] Darren A Hooks, Karl A Tomlinson, Scott G Marsden, Ian J LeGrice, Bruce H Smaill, Andrew J Pullan, and Peter J Hunter. Cardiac microstructure implications for electrical propagation and defibrillation in the heart. Circulation Research, 91(4):331-338, 2002.

[15] Robert H Jones, Eric J Velazquez, Robert E Michler, George Sopko, Jae K Oh, Christopher M O'Connor, James A Hill, Lorenzo Menicanti, Zygmunt Sadowski, Patrice Desvigne-Nickens, et al. Coronary bypass surgery with or without surgical ventricular reconstruction. New England Journal of Medicine, 360(17):1705$1717,2009$.

[16] Walter F Kerwin, Elias H Botvinick, J William OConnell, Scot H Merrick, Teresa DeMarco, Kanu Chatterjee, Kim Scheibly, and Leslie A Saxon. Ventricular contraction abnormalities in dilated cardiomyopathy: effect of biventricular pacing to correct interventricular dyssynchrony. Journal of the American College of Cardiology, 35(5):1221-1227, 2000. 
[17] Michael A Laflamme, Kent Y Chen, Anna V Naumova, Veronica Muskheli, James A Fugate, Sarah K Dupras, Hans Reinecke, Chunhui Xu, Mohammad Hassanipour, Shailaja Police, et al. Cardiomyocytes derived from human embryonic stem cells in pro-survival factors enhance function of infarcted rat hearts. Nature biotechnology, 25(9):1015-1024, 2007.

[18] Michael A Laflamme and Charles E Murry. Regenerating the heart. Nature biotechnology, 23(7):845-856, 2005.

[19] Denis Le Bihan and E Breton. Imagerie de diffusion in-vivo par résonance magnétique nucléaire. Comptes-Rendus de l'Académie des Sciences, 93(5):2734, 1985.

[20] Orsay Lycee Blaise Pascal. Tpe - l'irm.

[21] RICHARD J McCormick, TIMOTHY I Musch, BRYAN C Bergman, and D PAUL Thomas. Regional differences in lv collagen accumulation and mature cross-linking after myocardial infarction in rats. American Journal of Physiology-Heart and Circulatory Physiology, 266(1):H354-H359, 1994.

[22] Dariush Mozaffarian, Emelia J Benjamin, Alan S Go, Donna K Arnett, Michael J Blaha, Mary Cushman, Sandeep R Das, Sarah de Ferranti, JeanPierre Després, Heather J Fullerton, et al. Heart disease and stroke statistics2016 update a report from the american heart association. Circulation, pages CIR$0000000000000350,2015$.

[23] C. Carl Jaffe Patrick J. Lynch. (creative commons) medical image dataset. 2006.

[24] E. Piuze, J. Sporring, and K. Siddiqi. Maurer-cartan forms for fields on surfaces: Application to heart fiber geometry. IEEE Transactions on Pattern Analysis and Machine Intelligence, 37(12):2492-2504, Dec 2015.

[25] Emmanuel Piuze. The geometry of cardiac myofibers. PhD's thesis, 2014.

[26] Mihaela Pop, Nilesh R Ghugre, Venkat Ramanan, Lily Morikawa, Greg Stanisz, Alexander J Dick, and Graham A Wright. Quantification of fibrosis in infarcted swine hearts by ex vivo late gadolinium-enhancement and diffusion-weighted mri methods. Physics in medicine and biology, 58(15):5009, 2013.

[27] Mihaela Pop, Maxime Sermesant, Garry Liu, Jatin Relan, Tommaso Mansi, Alan Soong, Jean-Marc Peyrat, Michael V. Truong, Paul Fefer, Elliot R. McVeigh, Herve Delingette, Alexander Dick, Nicholas Ayache, and Graham A. 
Wright. Construction of 3d MR image-based computer models of pathologic hearts, augmented with histology and optical fluorescence imaging to characterize action potential propagation. Medical Image Analysis, 16(2):505-523, 2012.

[28] Michael JD Powell. The bobyqa algorithm for bound constrained optimization without derivatives. Cambridge NA Report NA2009/06, University of Cambridge, Cambridge, 2009.

[29] Frank E Rademakers, Walter J Rogers, William H Guier, Grover M Hutchins, Cynthia O Siu, Myron L Weisfeldt, James L Weiss, and Edward P Shapiro. Relation of regional cross-fiber shortening to wall thickening in the intact heart. three-dimensional strain analysis by nmr tagging. Circulation, 89(3):1174-1182, 1994.

[30] Damien Rohmer, Arkadiusz Sitek, and Grant T Gullberg. Reconstruction and visualization of fiber and laminar structure in the normal human heart from ex vivo diffusion tensor magnetic resonance imaging (dtmri) data. Investigative radiology, 42(11):777-789, 2007.

[31] Ulrik Sartipy, Anders Albåge, and Dan Lindblom. The dor procedure for left ventricular reconstruction. ten-year clinical experience. European journal of cardio-thoracic surgery, 27(6):1005-1010, 2005.

[32] Peter Savadjiev, Gustav J Strijkers, Adrianus J Bakermans, Emmanuel Piuze, Steven W Zucker, and Kaleem Siddiqi. Heart wall myofibers are arranged in minimal surfaces to optimize organ function. Proceedings of the National Academy of Sciences, 109(24):9248-9253, 2012.

[33] Leslee J Shaw, James K Min, Rory Hachamovitch, Eric D Peterson, Robert C Hendel, Pamela K Woodard, Daniel S Berman, and Pamela S Douglas. Cardiovascular imaging research at the crossroads. JACC: Cardiovascular Imaging, 3(3):316-324, 2010.

[34] Albert J Sinusas, Frank Bengel, Matthias Nahrendorf, Frederick H Epstein, Joseph C Wu, Flordeliza S Villanueva, Zahi A Fayad, and Robert J Gropler. Multimodality cardiovascular molecular imaging, part i. Circulation: Cardiovascular Imaging, 1(3):244-256, 2008.

[35] Kunhua Song, Young-Jae Nam, Xiang Luo, Xiaoxia Qi, Wei Tan, Guo N Huang, Asha Acharya, Christopher L Smith, Michelle D Tallquist, Eric G Neilson, et al. 
Heart repair by reprogramming non-myocytes with cardiac transcription factors. Nature, 485(7400):599-604, 2012.

[36] E. O. Stejskal and J. E. Tanner. Spin diffusion measurements: Spin echoes in the presence of a timedependent field gradient. The Journal of Chemical Physics, 42(1):288-292, 1965.

[37] Daniel D Streeter, Henry M Spotnitz, Dali P Patel, John Ross, and Edmund H Sonnenblick. Fiber orientation in the canine left ventricle during diastole and systole. Circulation research, 24(3):339-347, 1969.

[38] Bernard Swynghedauw. Molecular mechanisms of myocardial remodeling. Physiological reviews, 79(1):215-262, 1999.

[39] Philip C Ursell, Phyllis I Gardner, Arline Albala, JJ Fenoglio, and Andrew L Wit. Structural and electrophysiological changes in the epicardial border zone of canine myocardial infarcts during infarct healing. Circulation Research, 56(3):436-451, 1985.

[40] W. Vetterling W. Press, S. Teukolsky and B. Flannery. Numerical recipes 3rd edition: The art of scientific computing. Cambridge university press, 2007.

[41] Harlan F Weisman, David E Bush, John A Mannisi, and Bernadine Healy Bulkley. Global cardiac remodeling after acute myocardial infarction: a study in the rat model. Journal of the American College of Cardiology, 5(6):1355-1362, 1985.

[42] Nicolas Wiest-Daesslé, Sylvain Prima, Pierrick Coupé, Sean Patrick Morrissey, and Christian Barillot. Rician noise removal by non-local means filtering for low signal-to-noise ratio mri: applications to dt-mri. In Medical Image Computing and Computer-Assisted Intervention-MICCAI 2008, pages 171-179. Springer, 2008.

[43] Ed X Wu, Yin Wu, John M Nicholls, Jie Wang, Songyan Liao, Shuguang Zhu, Chu-Pak Lau, and Hung-Fat Tse. Mr diffusion tensor imaging study of postinfarct myocardium structural remodeling in a porcine model. Magnetic resonance in medicine, 58(4):687-695, 2007.

[44] Wolfram-Hubertus Zimmermann, Ivan Melnychenko, and Thomas Eschenhagen. Engineered heart tissue for regeneration of diseased hearts. Biomaterials, 25(9):1639-1647, 2004. 